\title{
2 Kommunikation durch die Ausstellung: Theorie
}

Die Erforschung der Kommunikation durch die Ausstellung kann auf einer Vielzahl von theoretischen wie empirischen Arbeiten aufbauen, die sich dem Phänomen der Ausstellungskommunikation aus unterschiedlichen Disziplinen genähert haben.

Anknüpfungspunkte bieten einerseits Arbeiten, die sich unmittelbar dem Thema Ausstellungskommunikation widmen, andererseits Arbeiten, die zwar nicht die Kommunikation durch die Ausstellung zum Thema haben, sich aber mit einem ihrer charakteristischen Merkmale beschäftigt haben, die wir im vorigen Kapitel herausgearbeitet haben. Der folgende Forschungsüberblick folgt im Wesentlichen dieser Zweiteilung $(2.1,2.2)$. Überschneidungen sind allerdings nicht selten: etwa wenn museologische Arbeiten zur Bedeutung von Museumsobjekten auf allgemeine semiotische Theorien zur Objektbedeutung zurückgreifen oder wenn eine linguistische Arbeit die zur Beschreibung multimodaler Zeichenarrangements entwickelten Kategorien auf die Analyse von Ausstellungsräumen anwendet.

In 2.1 gebe ich zunächst einen Überblick über Versuche, die Ausstellung als Ganzes unter dem Gesichtspunkt von Kommunikation zu betrachten (2.1.1). Dann referiere ich nacheinander die Forschung zu Museumsobjekten (2.1.2) und Museumstexten (2.1.3) sowie zu Ausstellungsdesign und -architektur (2.1.4), die gemeinhin als die drei grundlegenden Komponenten aufgefasst worden sind, die zur Konstruktion der Ausstellungsbedeutung genutzt werden. In den restlichen Abschnitten des Kapitels (2.2) stelle ich die linguistische und linguistikaffine Forschung zu den grundlegenden Merkmalen der Kommunikation durch die Ausstellung dar: Multimodalität (2.2.1), Raumgebundenheit (2.2.2) und Wissenskommunikation (2.2.3).

\subsection{Die Museumsperspektive}

\subsubsection{Ausstellung und Kommunikation}

Die Museumsausstellung ist das zentrale Kommunikationsmittel des Museums; mit ihrer Hilfe kommunizieren die Museumsverantwortlichen mit den Museumsbesuchern. Dieses Verständnis der Ausstellung, das wir so oder ähnlich formuliert in nahezu allen aktuellen Einführungen in die Museologie oder die Ausstellungsgestaltung finden können, ist nicht neu. Schon in den 1970er Jahren gibt es Arbeiten, die die kommunikativen Grundlagen der Ausstellung zum Thema machen (z.B. Kováč 1979), und Anfang der 1980er widmet sich eine Forschungs- 
tagung des ICOM, des Internationalen Museumsrats, exklusiv den kommunikativen Aspekten der Museumsausstellung (Sofka 1991). Trotz alledem beklagt Kaiser noch im Jahr 2006, dass der Museologie die notwendigen Grundlagen für das Verständnis und die Analyse der Ausstellungskommunikation fehlen:

\footnotetext{
Die Studien im Bereich Museumskommunikation und Ausstellungssprache zählen zu den jungen Forschungsgebieten der Museologie. Obwohl Ausstellungen zu den wichtigen Foren der Vermittlung von Kultur zählen, haben bislang weder Fach- noch öffentliche Diskurse adäquate Instrumente entwickelt, um Ausstellungen als Medien zu verstehen, zu analysieren und zu kritisieren. Eine Forschungslücke besteht nach wie vor in einer differenzierten Analyse der Ausstellungssprache. (Kaiser 2006: 14)
}

Dass trotz der langen Beschäftigung mit der Ausstellungskommunikation kein überzeugendes Analyseinstrumentarium entwickelt worden ist, hat meines Erachtens mehrere Gründe.

Einer dieser Gründe ist, dass eine prinzipielle Klärung des Kommunikationsbegriffs aussteht - im Abschnitt 1.2.1 haben wir schon gesehen, wie eine ausschließlich am Bild der Angesichtskommunikation entwickelte Auffassung von Kommunikation ein Verständnis der Kommunikation durch die Ausstellung behindern kann. Ein weiterer Grund ist, dass kommunikations- und zeichentheoretische Begriffe und Modelle oftmals oberflächlich und fragmentarisch rezipiert worden sind (vgl. die Kritik in Horta 1991: 56). Das spiegelt sich in der großen terminologischen Uneinheitlichkeit wieder, mit der über die Ausstellung geschrieben wird. Ungewiss ist, ob die Ausstellung nun ein Medium (Bianchi 2016: 251, Scholze 2004: 12), ein Kanal (Locher 2004, im Titel), eine Sprache im Sinn von Langue (Bellaigue 1991: 23) oder Parole (Gluziński 1991), eine Mitteilung (Waidacher 2005: 121), ein rhetorischer Text (Jones 1999a, 1999b) oder eine Narration (Flacke 2016) ist. Diese Begriffsvielfalt steht einer Bestimmung der Kommunikation durch die Ausstellung als einem einheitlichen Untersuchungsgegenstand im Wege.

Ebenso problematisch sind Analogien zwischen sprachlicher Kommunikation und Ausstellungskommunikation, die immer wieder gezogen werden, ohne die Tragfähigkeit dieser Analogien im Detail zu überprüfen und ihren zuweilen problematischen Implikationen nachzugehen. Museumsobjekte werden mit Wörtern gleichgesetzt, Objektgruppen mit Sätzen, Museumsarchitektur mit Dialekten oder Ausstellungsstile mit Vokabularen (Maranda 1991). Hier hat der Rückgriff der Museologie auf Semiotik und Sprachwissenschaft meines Erachtens die Erforschung der Ausstellungskommunikation eher behindert als gefördert. Denn die plausibleren dieser Analogien lassen eine tiefer gehende Erforschung der Kommunikation durch die Ausstellung als überflüssig erscheinen, indem sie ein problemloses Verstehen der Ausstellungskommunikation vorspiegeln, und 
die abwegigeren unter diesen Analogien diskreditieren auch die ernsthafte Suche nach Unterschieden und Gemeinsamkeiten von Sprach- und Ausstellungskommunikation.

Als fruchtbarer für das Verständnis der Ausstellungskommunikation haben sich dagegen Versuche erwiesen, allgemeine Kommunikationsmodelle wie Shannon/Weaver (1949) an den Fall der Ausstellungskommunikation anzupassen. Beispiele sind etwa Cameron (1968), Schiele (1989), Šurdič (1990), Horta (1992), Hooper-Greenhill (1991), Schmolke (2002) oder Kaiser (2006). Die Übernahme und Modifikation der allgemeinen Kommunikationsmodelle lenkt den Blick darauf, dass das in der Ausstellung Sicht- und Lesbare Bestandteil eines umfassenderen Kommunikationsprozesses ist, zu dem mindestens auch Sender und Empfänger gehören (selbst wenn diese bei der Analyse des Ausstellungsraums nicht anwesend sind). ${ }^{1}$ Das gestattet es der Museumsforschung Fragen zu stellen wie die, wer denn genau als „Sender“ und wer als „Empfänger“ aufzufassen ist oder welche Rolle dem Besucher im Gesamt der Ausstellungskommunikation zukommt: Dekodiert er lediglich die in die Ausstellung eingeschriebene Mitteilung, ${ }^{2}$ ist er jemand, der nach der Rezeption der Ausstellung selbst kommunikativ tätig werden kann (und so einen evaluativen „feedback loop“ in Gang setzt, vgl. Cameron 1968) oder ist er - und das ist die hier vertretene Sicht - schon beim Ausstellungsbesuch ein aktiver Part, der die Bedeutung der Ausstellung im Verlauf seines Besuchs konstruiert (s. Hooper-Greenhill 1991, Bellaigue 1991, Schärer 1991, Spielbauer 1991).

Über die musealen Kommunikationsmodelle hat auch die Frage nach dem (oder den) Code(s) des Museums Eingang in die Untersuchung der Ausstellungskommunikation gefunden. Mit der Untersuchung der musealen Codes sind zum einen die Vielfalt der Ausdrucksressourcen, die in der Ausstellung zum Einsatz kommen, in den Blick gekommen (wie etwa Hortas umfassende semiotische Ausstellungsanalyse eindrücklich vorführt: Horta 1992), zum anderen die allgemeine Frage nach Bedingungen des Verstehens der Museumsausstellung und der Gestalt der musealen ,Mitteilung‘. Mit dem Rückgriff auf den Code-Begriff sind jedoch auch Probleme verbunden. Untersuchungen, die dem oder den Code(s) des Museums auf der Spur sind, stellen sich diese Codes meist als allgemein geteilte kulturelle Regelwerke vor, die je eine Ausdruckseinheit und eine Bedeutungseinheit einander zuordnen. Ausstellungen werden als konkrete Realisierungen eines allgemeinen musealen Signifikations- und Kommunikations-Systems

1 Kritisch zu den musealen Kommunikationsmodellen: Mattl (1995: 20).

2 So beschreibt etwa Spencer (2002: 374) den Museumsbesuch wie folgt: „[W]e are taking in the messages [...] the museum has prepared for us“ (meine Hervorhebung, W. K.). 
verstanden, und Ziel der Untersuchung der musealen Codes ist die Beschreibung dieses Systems, der ,Syntax‘ (Taborsky 1983) oder ,Sprache‘ des Museums. Mit dieser Fokussierung auf allgemeine Codierungs- und Decodierungsregeln ist das Funktionieren der Kommunikation durch die Ausstellung allerdings nur unvollständig zu beschreiben. Denn das Verstehen einer Ausstellung basiert ebenso auf lokalen Regelhaftigkeiten, die wir uns im Verlauf des einzelnen Museumsbesuchs erschließen. Dass ein roter Saurierumriss auf einer Vitrinenfront, der von einer Reihe schwarzer Saurierumrisse umgeben ist, die Saurierfamilie signalisiert, mit der wir es in dieser Vitrine zu tun haben (während die schwarzen uns zeigen, welche anderen Saurierfamilien uns noch im gleichen Saal erwarten), ergibt sich nicht aus einem allgemeinen Museumscode, der die Bedeutung von Farben und Umrissen festlegt, sondern - wie Mattl (1995: 21) es nennt, aus einer „lokalen, d.h. nur im gegebenen Raum gültigen Syntax“, die sich uns erschließt, sobald wir zwei oder drei dieser Vitrinen betrachtet haben (s.u. 3.5). Für die Untersuchung der Kommunikation durch die Ausstellung gilt es deshalb, den im Begriff der Codes enthaltenen System-bias zu überwinden. Anstelle eine Liste fixer Form-Bedeutung-Zuordnungen $\mathrm{zu}$ erstellen, gilt es danach $\mathrm{zu}$ fragen, was den Besuchern jeweils lokal zu ihrer Bedeutungskonstruktion zur Verfügung steht. Dazu ist es nötig, die Ausstellung nicht als Realisierung eines zugrundeliegenden Systems zu verstehen, sondern sie als komplexe Einheit des Gebrauchs ernst zu nehmen.

Schließlich gibt es eine ganze Reihe semiotischer und von der Semiotik inspirierter Ausstellungsanalysen. Ein oft zitierter Klassiker der semiotischen Ausstellungsanalyse ist Barthes' Untersuchung der Wanderausstellung La grande famille des hommes (Barthes 1970), in der der Autor die Mechanismen aufdeckt, mit denen in dieser Ausstellung das ahistorische Bild einer umfassenden Gemeinschaft aller Menschen erzeugt wird, die einer gemeinsamen, unveränderlichen conditio humana unterworfen sind. Klassisch ist auch die Analyse der Repräsentation der Aborigines im Western Australian Museum von Hodge und D'Souza (Hodge/D'Souza 1979), in der die Autoren im Detail den Verzerrungen nachgehen, die auftreten, wenn eine Ausstellung eine Kultur darzustellen versucht, die nicht die Kultur der Ausstellungsverantwortlichen ist.

Hooper-Greenhill (1991: 51) bemerkt kritisch zu diesen frühen semiotischen Annäherungen an die Ausstellungskommunikation, dass es jenen stärker um das Aufdecken eines „hidden curriculum“ oder einer „underlying ideological agenda“ gehe, als dass sie versuchten, das ,normale“ Funktionieren der Ausstellungskommunikation semiotisch zu erfassen. Hinzu kommt häufig eine auffallende Empiriearmut. So stützt sich Barthes' Analyse der Ausstellung La grande famille des hommes im Wesentlichen auf den Titel und die Benennung der verschiedenen Abteilungen dieser Ausstellung und Flochs - im Übrigen inspirierender - Beitrag 
zum Lausanner Fotografiemuseum (Floch 1996) gibt die geringe Rolle, die die empirische Beobachtung für ihn spielt, schon im Untertitel zu erkennen: „Interrogations d'un sémioticien avant de visiter le musée de l'Elysée de Lausanne” (meine Hervorhebung, W.K.).

Für die hier vorliegende Arbeit sind deshalb vor allem jüngere semiotische Arbeiten von Interesse, die Ausstellungen in ihrer ganzen multimodalen Breite untersuchen, etwa Jones’ detailreiche „rhetorische“ Analyse des HolocaustMemorial-Museums (Jones 1999b), Scholze (2004), die eine semiotische Typologie verschiedener Ausstellungstypen entwickelt, die von Mieke Bals Kulturanalyse geprägte Studie Muttenthaler/Wonisch (2006) oder eine Reihe von Arbeiten aus dem Umfeld des Systemfunktionalismus (s. etwa Stenglin 2009, mit weiterer Literatur). ${ }^{3}$

\subsubsection{Museumsobjekte}

Ein Gutteil der Forschung zu der Frage, wie sich mit Hilfe der Museumsausstellung Bedeutungen vermitteln lassen, beschäftigt sich mit den Objekten der Ausstellung. Der Grund dafür liegt in der allgemeinen Überzeugung, dass Objekte das „primäre Medium“ (Korff 1984: 89) der Museumskommunikation sind, ja dass in der Kommunikation mit Hilfe von Museumsobjekten das ,Alleinstellungsmerk$\mathrm{mal}^{\star}$ des Museums zu suchen ist (Kavanagh 1989: 119).

Unbestritten ist in der Museumsliteratur, dass Objekte als Zeichen fungieren können. ${ }^{4}$ Damit tritt erneut die Semiotik als Wissenschaft von den Zeichen ins Blickfeld der museologischen Forschung; aber auch andere Disziplinen haben sich mit der Bedeutung von Objekten (und der Frage, wie sie zu erschließen ist) beschäftigt: etwa die aus Sozialanthropologie und Archäologie erwachsene Material-Culture-Forschung (z.B. Shanks/Tilley 1987, Kavanagh 1989, Hodder 1994 oder Veit/Kienlin/Kümmel/Schmidt 2003), die Kulturgeschichte (Kohl 2003) oder die Psychologie (Moles 1972, Habermas 1996).

Behandelt wurden im Wesentlichen die folgenden Aspekte: Was bedeuten Objekte? Woher kommen diese Bedeutungen? Und schließlich: Funktionieren

\footnotetext{
3 Dass sich trotz der beträchtlichen Zahl von semiotischen und semiotisch inspirierten Arbeiten zur Ausstellungskommunikation bis heute keine eigentliche Museumssemiotik ausgebildet hat, belegt das durchgehende Fehlen dieses Schlagworts in semiotischen Handbüchern und Überblicksdarstellungen (vgl. Trifonas 2015 oder Posner/Robering/Sebeok 1997-2004).

4 Nöth (2000b: 527) verweist auf zwei grundlegende Auffassungen, eine „dichotomische“, nach der es zeichenhafte und nicht zeichenhafte Aspekte von Objekten gibt, und eine „pansemiotische“, nach der alle Aspekte des Objekts als Zeichen analysiert werden können.
} 
Objektzeichen wie Sprachzeichen? Jedem dieser Aspekte ist im Folgenden ein Unterabschnitt gewidmet.

\subsubsection{Die Bedeutung von Museumsobjekten}

Die Frage nach der Bedeutung von Museumsobjekten beinhaltet zwei Teilaspekte. Zum einen geht es darum, was Objekte generell bedeuten (können), zum anderen darum, welche Bedeutung Objekten in der Ausstellung haben können.

Zur Beantwortung der ersten Teilfrage hat sich die Museologie häufig auf Barthes’ Analyse des Systems der Mode (Barthes 1967) oder seine Ausführungen zur Semantik der Objekte (Barthes 1988a) gestützt, aber auch eigenständig allgemeine semiotische Begriffe wie Denotation oder Konnotation für die differenzierte Beschreibung von Objektbedeutungen nutzbar gemacht (z.B. Pearce 1989, 1992: 166-191 oder Horta 1992). In der Regel geht es bei diesen Analysen um Artefakte, also vom Menschen hergestellte Objekte. ${ }^{5}$

Folgende Besonderheiten der Objektbedeutung sind von der Forschung herausgearbeitet worden:

- Objekte haben eine ,primäre Bedeutung‘, die in ihrem ursprünglichen praktischen Gebrauch, in ihrem ursprünglichen Verwendungszweck liegt bzw. in den „Intentionen“, die ihnen „bereits im Zuge ihres Herstellungsprozesses [...] eingegeben worden“ sind (Kohl 2003: 121). Zusätzlich kann, wie (Barthes 1967: 269ff.) beschreibt, der Gebrauch des Objekts zu einem Zeichen werden, sodass beispielsweise ein Ballkleid ,gesellschaftlicher Empfang' bedeutet; und dieses Gebrauchs-Zeichen kann schließlich ,naturalisiert' werden, sodass der gesellschaftlich zugeschriebene Nutzen des Objekts als etwas erscheint, das in der ,Natur` des Objekts zu liegen scheint (,Ballkleider sind für Empfänge da [weil sie sich natürlich dazu eignen]').

- Bisweilen wird die Ansicht vertreten, dass schon einzelne Objekte das Äquivalent einer Aussage darstellen (so etwa Barthes 1988a: 194: Ein Revolver, den man in einem Film sehe, denotiere nicht einfach ,Revolver', sondern entspreche der Aussage ,Hier ist ein Revolver!').

- Und schließlich: Bei aller Semantisierung bleiben Objekte Objekte. Sie bieten etwas, das Mattl (1995: 17) einen „,Überschuss‘ an Erfahrungsmöglichkeiten“ nennt: Es gibt immer mehr an einem Objekt wahrzunehmen, als das, was es zum Erkennen seines Zeichengehalts braucht. ${ }^{6}$

5 Zur Definition von „Objekt“ und verschiedenen Klassen von Objekten: Moles (1972: 21ff.) oder Kohl (2003: 123-132), zu „Objekt vs. Artefakt“: Nöth (2000b).

6 Für Korff (so z.B. Korff 1995a) liegt in diesem letzten Punkt die Einzigartigkeit des Museumserlebnisses begründet. Das authentische Objekt eröffnet eine breite Palette an Wahrnehmungs- 
Die Materialität der Objekte ermöglicht etwas, das Assmann (1988) die „wilde Semiose“ genannt hat. ${ }^{7}$ Wenn wir ein Objekt als Zeichen nutzen, nehmen wir es nur noch als Zeichenträger wahr und ,blicken durch seine Materialität hindurch“ direkt auf die repräsentierte Bedeutung, während gleichzeitig seine Materialität gleichsam unsichtbar wird. Doch wenn wir uns dieser Materialität gewahr werden (durch eine Form des Sehens, die Assmann ,Starren' nennt), können aus ihr neue Bedeutungen generiert werden:

Wilde Semiose bringt die Grundpfeiler der etablierten Zeichenordnung zum Einsturz, indem sie auf die Materialität des Zeichens adaptiert und die Präsenz der Welt wiederherstellt. In jedem Fall erzeugt sie Unordnung im bestehenden Beziehungssystem der Konventionen und Assoziationen, sie stellt neue, unmittelbare Bedeutung her, sie verzerrt, vervielfältigt, sprengt bestehenden Sinn. (Assmann 1988: 239)

Diese Eigenschaft von Objekten ist aus Museumsperspektive bald zur Grundlage der Einzigartigkeit des Museumserlebnisses erklärt worden (so von Korff 1995a), bald zu einem Hindernis musealer Kommunikation (Hahn 2016: 17).

\subsubsection{Quellen der Objektbedeutung}

Woraus speist sich die Bedeutung von Objekten? Während manche Autoren davon ausgehen, dass Objekte Bedeutungen besitzen, die es zu erschließen gilt (Korff 1995a: 22: „Dinge sind Zeugen, die Informationen über Vergangenes zu geben imstande sind“), betonen andere, dass die Bedeutung von Museumsobjekten das Ergebnis einer Konstruktion sind, sei es durch die Gesellschaft (Taborsky 1990, Kavanagh 1989: 132), sei es durch das Ausstellungsteam und die Besucher (Schäfer 1989: 45).

In diesem Zusammenhang ist lange darüber gestritten worden, ob es Objektbedeutungen gibt, die sich den Sinnen unmittelbar erschließen, also ohne den ,Umweg، über die Kenntnis eines Codes oder einen begleitenden Erläuterungstext. Denn mit dieser „Anschaulichkeit“ des Museumsobjekts wird häufig die Einzigartigkeit der Museumserfahrung begründet (kritisch: Weschenfelder/ Zacharias 1992: 73-75, Steen 1995). Diese Diskussion blieb aber fruchtlos, weil sie sich weder auf die empirische Analyse einzelner Museumsobjekte stützte, die offengelegt hätte, welche Erfahrungen denn konkret durch die Betrachtung

möglichkeiten, die aus dem Betrachten eines Exponats deutlich mehr machen als ein bloßes ,Lesen' seiner Bedeutung im Ausstellungszusammenhang; Mattl sieht den Erfahrungsüberschuss eher als Problem für die Ausstellungsplaner, die damit umgehen müssen, dass die Besucher mehr (und anderes) an einem Exponat sehen können, als von ihnen intendiert war.

7 Vgl. auch Niewerth (2018: 38-49). 
dieses Objekts zu machen sind, noch auf Theorien und Konzepte der Objektwahrnehmung.

Gut erforscht sind dagegen die Bedeutungseffekte, die die Eingliederung eines Objekts in das Museum - die sogenannte Musealisierung - mit sich bringt. Der Philosoph Krzysztof Pomian in seinem Essay über das Sammeln (Pomian 1988), Michael Thompson in seiner Abfalltheorie (Thompson 1979, vgl. Fehr 1989) und Abraham Moles in seiner Beschreibung des Durchgangs durch die ,réserve du grenier“ (Moles 1972: 43, 95-111) kommen zu ganz ähnlichen Beschreibungen des Vorgangs der Musealisierung.

Die Musealisierung besteht jeweils aus einem Prozess der „De- und eine Rekontextualisierung“ (Kohl 2005: 35), in dem die Objekte ihren Warenwert verlieren, dem Warenkreislauf entzogen werden und schließlich durch den Einbezug in eine Sammlung einen neuen symbolischen Wert und neue Bedeutungen erlangen. In Pomians Begriffen werden „Dinge“ (über die Zwischenstation des „Abfalls“) zu „Semiophoren“. „Dinge“ sind

nützliche Gegenstände, [...] die konsumiert werden können oder die dazu dienen, sich Subsistenzmittel zu verschaffen oder auch Rohstoffe umzuwandeln, so daß sie konsumiert werden können oder schließlich dazu, gegen die Veränderungen der natürlichen Umgebungen zu schützen (Pomian 1988: 49)

„Semiophoren“ dagegen

Gegenstände ohne Nützlichkeit im ebenpräzisierten [sic] Sinn, sondern Gegenstände, die [...] mit einer Bedeutung versehen sind (ebd.).

Im Prozess der Musealisierung verlieren Objekte ,their original determined functions and conventional significations “ und ,,are open to all forms of use and constructions chosen by the museum emitters“ (Horta 1992: 34f.).

Die allererste Bedeutung, die Objekte durch die Musealisierung gewinnen, ist die, Museumsobjekt $\mathrm{zu}$ sein, eine Konnotation, die alle anderen Bedeutungen durchdringt (1992: 30). ${ }^{8}$ Eine zweite neue Bedeutung ergibt sich aus dem Platz, der dem Einzelobjekt in dem (von den einschlägigen Fachwissenschaften bestimmten) System der Sammlung zugewiesen wird (vgl. die „taxonomische Koordinate“ der Objektbedeutung bei Barthes 1988a: 191f.). Hierzu gibt es eine

8 Kohl (2005: 33) macht auf einen interessanten Unterschied zu sakralen Räumen aufmerksam: Erst die sakralen Gegenstände (z. B. Reliquien) machen Tempel oder Kirchen zu heiligen Orten. Im Museum ist das umgekehrt: Museumsobjekte erhalten erst durch ihre Präsentation im Museum eine besondere Aura. 
lange praktische Tradition der Bestimmung von Objektbedeutungen zur Katalogisierung und Systematisierung von Sammlungen (s. etwa Elliot et al. 1994 oder Pearce 1994).

Was die weiteren Bedeutungen angeht, die mit einem Museumsobjekt im Rahmen einer Ausstellung vermittelt werden können, sind die Möglichkeiten nahezu unbegrenzt. Jedes Objekt kann, wie Mattl (1995: 18) herausstellt: „zum Repräsentanten einer ganzen Klasse oder auch - so paradox es klingt - ausschließlich seiner selbst aufsteigen.“ Dies illustriert Freydank (1990) sehr eindrücklich am Beispiel der Dermoplastik eines Sibirischen Tigers aus dem Museum für Naturkunde der Humboldt-Universität, die in verschiedenen Ausstellungen zum Einsatz kam. Einmal repräsentierte sie die Gattung Panthera tigris, einmal illustrierte sie ein biologisches Gesetz, nach dem Tiere in kälteren Weltregionen größer sind als in wärmeren, einmal stand sie stellvertretend für Bemühungen um die Rettung aussterbender Tiere in Russland, und einmal schließlich illustrierte sie - als ,Repräsentant ihrer selbst“ - die Geschichte der Tierpräparation. ${ }^{9}$

Typische Relationen zwischen Museumsobjekten und ihren Bedeutungen sind mit den rhetorischen Begriffen Metonymie und Metapher erfasst worden (etwa von Šurdič 1990 oder Müller-Scheessel 2003). Bei der metonymischen Verwendungsweise steht das Museumsobjekt für etwas, von dem es selbst ein Teil ist (so z. B. der Kapitelstein für den Kirchenbau, in den dieser Stein eingefügt war), bei der metaphorischen Verwendungsweise steht es für etwas, zu dem es in einer beliebigen Kontiguitätsbeziehung steht (im Fall des Kapitelsteins: Religion, den Beruf des Steinmetzen usw.). Auch können Objekte zu dem, was sie repräsentieren, in einer Relation der Exemplifizierung stehen. Diese Relation erläutert Goodman (1968: 529) am Beispiel eines Stoffmusters beim Möbelhändler, das einen bestimmten Stoff repräsentiert, den man bestellen kann, gleichzeitig aber aus diesem Stoff besteht. ${ }^{10}$ Im Fall der Exemplifizierung, so Goodmans Beschreibung, sind Gegenstand und Verweisobjekt aus der gleichen Ausdruckssubstanz, wobei das Muster das Produkt denotiert, während gleichzeitig das Produkt das Muster determiniert, und zwar in dem Sinne, dass es festlegt, was an dem Muster die ,bedeutsamen` Eigenschaften sind.

\footnotetext{
9 Eindeutig zu kurz greift deshalb Kohls Behauptung eines generellen Bedeutungseffekts der Musealisierung: „Durch die Präsentation in der Ausstellung erhalten Objekte den Charakter von ,Prototypen““, die „Reihen [bilden], in denen eines durch ein anderes ersetzt werden kann, ohne daß sich dadurch der semantische Gehalt grundsätzlich ändert“ (Kohl 2003: 255).

10 Dazu Nöth (2000b: 526): „Zeigegegenstände sind nach dieser Beschreibung also in gewisser Weise Zeichen(träger) und ihr eigenes Objekt zugleich“.
} 
Wie Museumsobjekte in der Ausstellung interpretiert werden, hängt - wie wir in Kapitel 3 sehen werden - ganz wesentlich von ihrer Platzierung im Ausstellungskontext ab. Doch gibt es zu der Frage, wie der Kontext konkret die Bedeutung eines Exponats beeinflusst und welche Bedeutungen auf welche Weise durch die Anordnung von Objekten vermittelt werden können, bisher kaum Untersuchungen. Zwar ist vereinzelt von der ,Grammatik' der Museumssprache die Rede, aber damit ist meist eine bloße Auflistung der vielfältigen Ausdrucksmöglichkeiten im Museum gemeint (s. beispielsweise Hall 1987, die das Wort grammar im Untertitel führt). Die Syntaktik, die Beschreibung der Regeln und Effekte der Kombination von Museumsobjekten (und Elementen anderer, in der Ausstellung präsenter Zeichensysteme) wird selten und nie systematisch untersucht.

Das mag daran liegen, dass es auch außerhalb der Forschung zu Museumsausstellungen nur wenig Überlegungen zur Syntaktik der Objekte gibt. So beschränken sich Barthes’ Überlegungen zur Syntaktik von Objekten im Wesentlichen auf die verkürzende Behauptung, Objekte stünden lediglich in einer Relation der Parataxe zueinander (Barthes 1988a: 194). Doch gibt es im Raum sicher nicht nur ein additives Nebeneinander von Objekten, sondern auch topologische Relationen des Über- und Untereinander, des Einschlusses, der Durchdringung, der Verdeckung usw.

Ausführliche Gedanken zur Syntaktik der Objekte findet man meines Wissens lediglich in Moles' sozialpsychologischer Théorie des Objets (Moles 1972). Der Autor sucht nach „les modes d'assemblage, la syntaxe des objets ensemble à l'intérieur du discours constitué par le display quotidien“ (1972: 113). Moles’ Gesetze der Anordnung von Objekten im Raum scheinen mir aber zu heterogen und ihre Zusammenstellung zu willkürlich zu sein, um sich als Grundlage für eine systematische Untersuchung der räumlichen Anordnung der Ausstellung zu eignen. Sie reichen von ,Naturgesetzen“ wie dem der „contrainte volumique“ (1972: 115: Die Summe der Volumen der Objekte in einem Raum muss kleiner sein als das Gesamtvolumen) über solche, die sich aus kulturellen Konventionen ergeben (,densité optimum“: Wo im Raum soll die größte Objektdichte herrschen? 1972: 115) bis hin zu schwer operationalisierbaren ,Gesetzen', wie dem des „rayonnement de l'objet (1972: 116), wonach jedes Objekt eine Einflusssphäre hat, die nicht unterschritten werden darf, weil sich die Objekte sonst ,stören'.

Meines Wissens existiert nur eine einzige systematische Analyse des Zusammenhangs zwischen musealen Präsentationsformen und der Bedeutung von Museumsobjekten: Shanks/Tilley (1987: 68-90). Die Autoren beschreiben, wie Museumsobjekte als Güter im akademischen ,Warenaustausch' erscheinen können, mit Objektkennungen als ihren „academic price-tags“ (1987: 69), aber auch als ästhetische Objekte, als Produkte, die die Fähigkeiten des Homo artifex, des Menschen als Künstler und Handwerker, feiern 1987: 73). In anderen Ausstel- 
lungszusammenhängen („narrative displays“, 1987: 74-76) ist das Objekt Beleg für eine mit Hilfe von Kontextinformationen geschaffene ,Erzählung،, und in wieder anderen schließlich (,situational displays“, 1987: 76-79) Teil einer naturalistischen Inszenierung, in der die kommunikative Funktion der Objekte im Vordergrund steht. ${ }^{11}$

Weitere Hinweise auf Bedeutungen, die sich aus dem Zusammenhang der Präsentation ergeben, lassen sich indirekt aus Untersuchungen gewinnen, die heutige wie historische Ordnungsprinzipien von Museumsausstellungen zu rekonstruieren und zu klassifizieren versuchen. Scholze (2004) etwa beschreibt anhand moderner Dauerausstellungen vier Ordnungsprinzipien: Neben Klassifikation und Chronologie sind das Inszenierung und Komposition (wobei mit Letzterem eine ästhetisch anspruchsvolle ,Mischung 'von Objekten gemeint ist). Speziell der Inszenierung als Ordnungsprinzip ist Kaiser (2006) gewidmet. Die Autorin unterscheidet zwei „Raumbilder“, wie sie die räumlichen Resultate von Inszenierungen nennt: „rekonstruierende“ oder „abstrahierende“ Raumbilder (2006: 38-46, dort auch Literatur zu alternativen Einteilungen). Erstere sind „all die Inszenierungen, die zum Ziel haben, eine bestimmte historische Begebenheit zu rekonstruieren oder an historische Situationen zu erinnern“ (2006: 40), letztere dagegen solche Formen der Inszenierung, die einen künstlerischen, ästhetisierenden, auf das Erlebnis ausgerichteten Umgang mit den Objekten pflegen und bei der die Fachwissenschaft in den Hintergrund tritt. Einen guten geschichtlichen Überblick über die Karriere des Inszenierungsbegriffs im Museumskontext gibt Baur (2016).

Historische Studien zur Früh- und Vorzeit der Museen benennen als mögliche Ordnungsprinzipien:

- die Anordnung nach den Reichen der Natur (z.B. Becker 1994: 33-35),

- die Anordnung nach der Ähnlichkeit der Materialien (s. MacGregor 1994: 94, 97),

- die unterschiedlichen Grade von „Kuriosität“ und „Rarität“ der ausgestellten Gegenstände (Kristensen 1994: 132f.),

- die Anordnung entsprechend einer begrifflichen Taxonomie (Felfe 2006: 13)

- die Anordnung als Tableau (das Wissen als statische Struktur) vs. als Parcours (die chronologische Ordnung, Foucault 1974: 46-77).

Die Einbettung des einzelnen Museumsobjekts in derartige räumliche Zusammenhänge hat, wie wir in Kapitel 3 sehen werden, wichtige Konsequenzen für

11 Ein Ausstellungsexperiment, das diesen und ähnlichen Bedeutungseffekten durch den Kontext der Präsentation nachgeht, wird in Schärer (1991) beschrieben. 
die Konstruktion der Bedeutung des einzelnen Objekts, aber auch für die Interpretation eines räumlichen Nebeneinanders: als ein konzeptuelles Nebeneinander in einer Taxonomie oder Meronymie, ein zeitliches Nacheinander in einer ,Erzählung‘ oder Chronologie, ein räumliches Nebeneinander in einem ,zeitlich verschobenen' Wahrnehmungskontext oder ein konzeptuelles Nebeneinander in einem künstlerischen Objektarrangement im Raum.

\subsubsection{Objektzeichen und Sprachzeichen}

Um die Eigenheiten der Museumsobjekte beschreiben zu können, hat man häufig nach allgemeinen Unterschieden zwischen objektbasierten Zeichensystemen und dem Sprachsystem gesucht. Dabei wurden folgende Unterschiede herausgearbeitet (vgl. Hahn 2003 oder Frerichs 2003):

- Objekte sind prinzipiell polysemisch. Waidacher (1999: 255) etwa nennt das Objekt „eine Art visuelle Kurzschrift“, die Raum für Deutungen lasse. Tatsächlich sind aber auch Sprachzeichen polysemisch. Oft erscheint uns ihre Bedeutung nur eindeutig zu sein, weil wir routinehaft und weitgehend unbewusst den Ko- und Kontext des sprachlichen Zeichens genutzt haben, um die Bedeutung des Sprachzeichens zu vereindeutigen.

- Objektbedeutungen sind weniger scharf umrissen, auch weil sich im Objekt die Primärbedeutung, Bedeutungen, die durch die Musealisierung hervorgebracht worden sind, und Bedeutungsanteile aus der Materialität des Objekts überlagern oder im Widerstreit befinden können (s.o.). Allerdings sei daran erinnert, dass Assmanns ,wilde Semiose“ nicht auf Objektzeichen beschränkt ist: In 2.2.1 werden wir sehen, dass auch die Materialität der Schrift zum Träger eigenständiger Bedeutungen werden kann.

- Für die Interpretation von Objekten ist der Kontext oft wichtiger als die Kenntnis eines Codes. Dies gilt sicher nicht für alle Objektzeichen. Zweifelsohne gibt es Objektzeichen, für die es - ebenso wie für Sprachzeichen - Bedingungen der Wohlgeformtheit gibt und eine „community of users“, die diese Bedingungen kennt (vgl. McNeill 1992: 38f.).

- Systeme von Objektzeichen sind nicht, doppelt gegliedert‘. Es ist also nicht möglich, Objekte so zu segmentieren, dass sich minimale Ausdruckseinheiten ergeben, die Bedeutungen unterscheiden, selbst aber keine Bedeutung besitzen. Damit steht die Beobachtung im Zusammenhang, dass sich Objektbedeutungen nicht aus den Bedeutungen kleinerer Einheiten zusammensetzen, also nicht dem Kompositionsprinzip gehorchen; Objekte bedeuten vielmehr ,en bloque،, als Gesamtheit. Diese Behauptung zur Bedeutung einzelner Objekte mündet in Aussagen zu Arrangements aus mehreren Objekt- 
zeichen, denen - wie wir im letzten Abschnitt gesehen haben: zu unrecht - eine auf die Parataxe beschränkte Syntax nachgesagt wird.

- Die Ausdrucksmöglichkeiten von Objekten sind im Vergleich zur Sprache beschränkt. Allein mit Objekten lassen sich keine Negationen ausdrücken, und Objekte allein erlauben keine Metakommunikation. Dieser Aspekt ist in der interdisziplinären Diskussion um das Bild in Bildwissenschaft, Bildlinguistik und Multimodalitätsforschung ausführlich behandelt worden (s. etwa Nöth 2004, 2016, Worth 1981, Machin 2007). ${ }^{12}$ Mit Hinblick auf die Ausdrucksmöglichkeiten von Museumsobjekten hat eine solche Debatte bisher nicht stattgefunden. Hier gibt es lediglich einige wenige Arbeiten zu den kommunikativen Funktionen von Museumsobjekten (etwa Šurdič 1990 oder Horta 1992: 116).

Zusammenfassend kann man festhalten, dass es eine breite, semiotisch inspirierte Forschung zur Bedeutung von Objekten (und speziell von Museumsobjekten) gibt, auf der die Untersuchung der Ausstellungskommunikation aufbauen kann. Allerdings gilt es dabei, die spezifische konstruktivistische Perspektive dieser Arbeit im Auge zu behalten.

Diese macht es notwendig, die Frage der Objektbedeutung konsequent von der Rezeption aus zu beantworten. Das heißt: die Analyse der Objektbedeutung muss von dem ausgehen, was den Besuchern im Moment der Begegnung mit dem Objekt alles für ihre Bedeutungskonstitution zur Verfügung steht: all das, was sie im Moment der Rezeption wahrnehmen, lesen oder ausgehend von dem Gesehenen und Gelesenen an Verstehenskontexten aktivieren können. Das hat weitreichende Folgen für die Analyse. Die relevante Analysefrage lautet dann nämlich, welche kommunikativen Erscheinungsformen dafür verantwortlich sind, dass ein bestimmtes Objekt als etwas Natürliches oder etwas Menschengemachtes (ein Artefakt), als Gegenstand wissenschaftlicher Forschung (also als OriginalDatum) oder als Objekt der musealen Vermittlung (Exponat) verstanden wird. Wie Shanks/Tilley (1987: 68-90) gezeigt haben, entscheiden nicht Eigenschaften

12 Bestritten worden ist im Einzelnen, dass Bilder in der Lage seien, auf sich selbst zu referieren oder sich zu kommentieren (nach Worth 1981: 179 haben sie „no ability to make ,meta“ statements about lower-level statements of the picture system. A picture cannot comment on itself. A picture cannot depict ,This picture is not the case“ or ,This picture is not true“"); dass Bilder „conditionals, counterfactuals [...] or past-future tenses“ ausdrücken können (Worth 1981: 178); dass sie semantische Relationen zwischen Aussagen formulieren können (z.B. ein oder oder ein weil, s. Machin 2007); dass Bilder überhaupt die Fähigkeit besitzen, Propositionen zu formulieren (Worth 1981: 178) - eine Behauptung, die zu Barthes’ Ansicht, Objektzeichen seien immer Aussagen (Barthes 1988) im Gegensatz steht. 
des Objekts selbst darüber, wie diese Frage zu beantworten ist, sondern die Art der Präsentation. Auch die referierten Einsichten in die Bedeutungseffekte der Musealisierung müssen in der Rezeption verankert werden: die Analyse muss diejenigen kommunikativen Erscheinungsformen identifizieren, die es den Besuchern signalisieren, dass ein bestimmtes Objekt als Exponat im Rahmen von Ausstellungskommunikation genutzt werden soll (also: zum Betrachten, zum Lernen eingesetzt werden soll), und gerade nicht in seiner ursprünglichen Bedeutung und Verwendung. Weiterhin gilt es, die Erforschung der Beziehungen des Einzelobjekts zu seinem räumlichen Kontext zu erweitern und zu vertiefen, der - wie wir gesehen haben - die Bedeutung der Objekte maßgeblich beeinflussen kann. Damit ist nicht gemeint, dass eine allgemeine Grammatik der Raumbeziehungen formuliert werden soll (im Sinne von Moles',Gesetzen'). Vielmehr geht es darum zu fragen, wie im Raum signalisiert wird, welche Zusammenhänge zwischen Objekten - und anderen Zeichen im Raum - für die Konstruktion der Objektbedeutung herangezogen werden sollen.

\subsubsection{Texte im Museum}

Das zweite große Thema der museologischen Beschäftigung mit der Ausstellungskommunikation sind die Texte, die in der Ausstellung zu lesen sind.

Bemerkenswert scheint mir, dass Texte von der Museologie zuallererst als Alternative zu Objekten verstanden und diskutiert worden sind. Bis heute werden Texte häufig noch als Fremdkörper in der Ausstellung aufgefasst (so von Waidacher 2000: 4, der sie zu den „fremde[n] Kommunikationsformen“ zählt), und das, obwohl schon in einer Wunderkammer des 18. Jahrhunderts erklärende Schilder an Objekten belegt sind (MacGregor 1994: 74). Seit den 1970er Jahren wurde über die Berechtigung oder Notwendigkeit von Texten im Museum eine Jahrzehnte anhaltende, heftige Debatte geführt (unter sich wandelnden Etiketten: „Lernort oder Musentempel“, „Inszenierung vs. Text“; vgl. Steen 1995: 47f.). Im Kern geht es darum, ob sich Ausstellungen auf den kommunikativen Eigenwert ihrer Objekte verlassen können oder ob Objekte erst durch Texte eine Bedeutung erhalten bzw. verstehbar werden (vgl. etwa ebd. und Korff 1995b). Verfechter der Aussagekraft des ,authentischen Objekts“13 führen einen Feldzug gegen das „booklet spread out on the walls“ (Miller 1990: 86) oder das „2-D-syndrome“ (Tripps 1987)

13 Zur Originalität und Authentizität als grundlegender Kriterien für das Gelingen der museumsspezifischen Kommunikation s. Weschenfelder/Zacharias (1992: 76f.) oder Lord/Lord (2002: 16). Kritisch dazu Endrödi/Krefting (1989: 98). 
und sehen in Texten eine Konkurrenz zur ,Aura‘ der Museumsobjekte (s. dazu Korff 1995a: 24). Die Vertreter der Gegenposition halten dagegen, die Textgegner schüfen „Schatzkammern“, die „mit der Informationsverweigerung den Betrachter in geradezu undemokratischer Weise [...] entmündigen“ (Kaiser 2006: 18). Diese unterschiedlichen Positionen ergeben sich zweifellos aus unterschiedlichen disziplinären Traditionen im Umgang mit Objekten (Steen 1995).

Sieht man von den Beiträgen zu dieser Debatte ab, wird der Großteil der Literatur zu Texten im Museum von Ratgebern gebildet, die sich mit der praktischen Frage auseinandersetzen, wie, gute“ Texte im Museum zu verfassen sind (exemplarisch Serrell 2015).

Ein Aspekt ist hierbei sehr prominent, nämlich der Aspekt der Vermittlung von Wissen. Dabei geht es im Wesentlichen darum, wie Texte für Laien verständlich $\mathrm{zu}$ formulieren sind und wie Texte auf die Tatsache reagieren können, dass ihre Leser ganz unterschiedliche Wissensvoraussetzungen haben können. Aus linguistischer Sicht überraschend ist aber, dass die sprachlichen Techniken der Wissensvermittlung (etwa im Sinne der „Veranschaulichungsverfahren“ Brünner/ Gülich 2002, Brünner 2013 und s.u.; oder der in Niederhauser 1999 beschriebenen Strategien der Popularisierung) kaum je in den Blick geraten, geschweige denn im Detail behandelt werden. Andere charakteristische Merkmale von Ausstellungstexten bleiben nahezu unbeachtet, nämlich ihre Raumgebundenheit und ihre Einbettung in eine multimodale Umgebung.

Der Raumaspekt tritt immer wieder zutage, wenn Ratschläge zur Textgestaltung mit konkreten räumlichen Bedingungen der Ausstellungsrezeption begründet werden. Mit dem Verweis auf die unbequeme stehende oder gehende Rezeptionssituation werden Ratschläge zur Größe und Gestaltung der Schrift (Waidacher 2005: 165-173), zur Reduktion der Textlänge (z.B. Merleau-Ponty/ Ezrati 2005: 129f.) oder zum modularen, vom Wichtigen zum Unwichtigen fortschreitenden Textaufbau rechtfertigt (Zebhauser 2000: 24). Und Dawid/Schlesinger (2002: 42f.) begründen ihre Forderung nach einer klar signalisierten Textsortenhierarchie und einer strikten Kontrolle der Bezüge zwischen Saal- und Objekttexten damit, dass der Besucher sich die Bedeutung der Ausstellung nur Schritt für Schritt ausgehend von Einzeltexten erarbeiten kann. Systematische Überlegungen dazu, welche Bedeutungspotenziale durch die konkrete Position der Texte im Ausstellungsraum entstehen können, fehlen allerdings.

Noch seltener als auf den Aspekt der Raumgebundenheit bezieht sich die Ratgeberliteratur auf die Multimodalität der Ausstellungskommunikation, also auf die Frage, wie Texte mit den anderen Ausdrucksressourcen der Ausstellung in Beziehung zu setzen sind (eine Ausnahme ist Serrell 2015: 147-164). Dies beklagt auch Blunden (2020: 2): „[T] he semantic relations construed between a displayed object and its related verbiage remain largely unexamined“. Anstelle von kon- 
kreten Anweisungen zur Signalisierung solcher Beziehungen findet man in der Regel allgemeine Ratschläge, die an die oben erwähnte Text-oder-Objekt-Debatte anschließen (etwa: „Gute Ausstellungstexte funktionieren wie die Stimme aus dem Off bei einem Dokumentarfilm. Die Bilder stehen im Vordergrund“, Dawid/ Schlesinger 2002: 12).

Während sich an der Debatte um Notwendigkeit und Nutzen von Texten im Museum viele Autoren beteiligt haben und die Ratgeberliteratur umfangreich ist, gibt es auffallend wenig Arbeiten, die sich mit Texten in der Museumsausstellung auf der Grundlage empirischer Analysen auseinandersetzen.

Den größten Teil der empirischen Arbeiten zu Museumstexten versucht, Faktoren für deren Verständlichkeit (Literatur s. Coxall 1991: 86f.) oder noch grundlegender: für deren optische Lesbarkeit zu ermitteln (Wolf/Smith 1993, Borun/ Miller 1980 etc.). Gestellt werden Fragen wie die, wie viel Prozent der Besucher wie viele Texte lesen oder wie lange die Besucher der Lektüre eines Texts durchschnittlich widmen (vgl. Serell 2020 zum „sweep rate index“). Der Inhalt der Texte (ja selbst seine sprachliche Form) spielt in diesen Untersuchungen kaum je eine Rolle.

Arbeiten, die an Form und Inhalt der Texte interessiert sind, gibt es nur wenige. So haben sich (neben der schon erwähnten Arbeit von Barthes, Barthes 1970) auch Coxall (1994) und Purser (2000) der Aufdeckung von ,hidden agendas“ in Museumstexten gewidmet: Coxall (1994) macht darauf aufmerksam, dass Texte immer auch das Wertesystem ihrer Verfasserinnen und Verfasser vermitteln, und Purser (2000), die die Darstellung der Kultur australischer Aborigines im Ethnologischen Museum in Dahlem untersucht, weist im Vitrinentext sowie in einem Mitnahmeblatt eine verzerrte Darstellung der Aborigines-Kultur nach.

Eng am Text arbeiten auch Horta (1992) und Ventola/Hofinger (2004), allerdings ohne dezidiert kritische Perspektive. Horta (1992) untersucht - neben vielen anderen Ausdrucksressourcen - auch die Texte in einer Buddhismusausstellung und kann dabei zeigen, wie in diesen Texten eine kunsthistorische und eine religiöse Perspektive auf die Ausstellungsstücke aufeinandertreffen und welche Probleme sich daraus ergeben. Ventola/Hofinger (2004) untersuchen ein Bild in einem Saal des Mozart-Wohnhauses in Salzburg und den dazugehörigen Audioguide-Text und zeigen auf, wo sich Text und Bild in ihrer Aussage unterstützen und wo es Unterschiede in Text- und Bildkomposition gibt.

Bradburne (2000) schließlich identifiziert ausgehend von konkreten Textbeispielen allgemeine „user languages“, durch die die Interaktionsmöglichkeiten der Besucher auf je spezifische Weise geformt und beschränkt werden (2000: 26-30). In der ,Benutzersprache“ der „textual authority“ weist sich der Text als Stimme der Autorität aus, die die einzig richtige Interpretation der Museumsdinge liefert. In der Sprache der „observation“ werden den Besuchern die Dinge zur Beobach- 
tung unterbreitet und ihnen so eine ,eigene Autorität' als Beobachter zugewiesen. In der Sprache der „variables“ werden die Besucher aufgefordert, die unsichtbaren Beziehungen zwischen den Dingen zu entdecken, in der Sprache der „problems“ wird ihnen ein Rätsel gestellt usw. (2000: Kap. 4). ${ }^{14}$

Zusammenfassend kann man sagen, dass Analysen von Ausstellungstexten, die deren Inhalt und deren konkrete sprachliche Form ernst nehmen, bisher Mangelware sind. Konkret geht es darum, wie ein Text eine bestimmte Lesart oder eine bestimmte Nutzung eines Exponats nahelegen kann, wie durch mehrere Texte thematische Zusammenhänge gebildet werden können, wie sie die Konstruktion von Wissen ermöglichen usw. Auch dem Aspekt der Raumgebundenheit der Ausstellungstexte (und damit: ihrer Einbettung in eine hochgradig multimodale Umwelt) wurde bisher noch kaum Aufmerksamkeit zuteil. Im Gegenteil: Texte werden - wie in dem folgenden Zitat - häufig nicht als vollgültiger Bestandteil der Ausstellung verstanden:

Wenn hier und im Weiteren von Ausstellungsinhalten geredet wird, sind nur bedingt Informationen mündlicher oder schriftlicher Art gemeint, Ausstellungen werden vielmehr als Medien verstanden, die in erster Linie durch räumliche und visuelle Erfahrungen mitteilen und bedeuten. (Scholze 2004: 12, Fn. 9)

Wie schon im Fall der Objekte ist es notwendig, bei der Analyse der Ausstellungstexte von der Situation der Rezeption auszugehen (und das heißt: inklusive aller bei der Rezeption zur Verfügung stehenden Wahrnehmungen). In dieser Hinsicht geben die Arbeiten, die im letzten Absatz erwähnt worden sind, wichtige Impulse für die Analyse. Diese empirischen Studien lenken den Blick auf die multimodale Vielfalt der Zeichen, die dem Besucher im Ausstellungsraum für die Konstruktion der Ausstellungsbedeutung(en) zur Verfügung stehen und zeichnen detailliert nach, welche sprachlichen Formen welche Lesarten oder Nutzungsweisen des Ausstellungsraums fördern.

14 Andere Kategorien entwickelt Horta (1992: 154-156), die in Ausstellungstexten unterschiedliche Erzählerpositionen identifiziert, die sie nach Todorov „'aspects“ of the narrative“ nennt: „,the vision from behind“ (der Text vermittelt Deutungen oder Erläuterungen), „the vision with“ (der Autor verschwindet ganz hinter der Darstellung), „the vision from outside“ (der Autor beschreibt, was zu sehen ist). 


\subsubsection{Ausstellungsdesign und Architektur}

Ausstellungsarchitektur und -design werden häufig neben Objekten und Sprache als der dritte große Code der Ausstellungskommunikation aufgefasst (s. etwa Horta 1992, Jones 1999b), und die Relevanz des Raums für die Konstruktion der Ausstellungsbedeutung wird häufig betont (vgl. das Zitat von Scholze im letzten Abschnitt). In Anbetracht dieser Tatsache ist es überraschend, wie wenig Konkretes man aus der Literatur über die kommunikative Leistung von Museumsarchitektur und Ausstellungsdesign erfahren kann. ${ }^{15}$

Zwar ist die kommunikative Relevanz des Ausstellungsdesigns ${ }^{16}$ seit den 1980er Jahren verstärkt zum Thema der Forschung geworden, nachdem sie bis dahin, wie Staniszewski schreibt, „officially and collectively forgotten“ war (Staniszewski 1998: xi). Einige Essays und wissenschaftliche Texte haben seitdem zu ergründen versucht, welche Bedeutungen oder ideologischen Inhalte beispielsweise durch Hängungen, Raumgestaltung usw. transportiert werden können: etwa O’Dohertys bekannter Essay zur Präsentationsform der „weißen Zelle“ (O’Doherty/Kemp 1996), Staniszewski (1998) zur Entwicklung der Präsentationsformen moderner Kunst im 20. Jahrhundert oder Newhouse (2005) zur Präsentation altägyptischer Objekte als Kunstobjekte vs. als archäologische Gegenstände. Und immer wieder wird betont, das Ziel des Ausstellungsdesigns sei es,

die komplexen Inhalte anschaulich und begreifbar zu machen, die Bedeutung der Originale zu verstärken und damit die wissenschaftlich erarbeiteten Aussagen der Ausstellung zu vermitteln. (Teufel 2001: 13)

Schon im Jahr 1939 schrieb der Ausstellungsgestalter und Bauhauslehrer Herbert Bayer (in experimenteller Kleinschrift):

in exhibition design, we have a new and complex means of communication of the idea [...]. all the elements suited to the purpose of communicating the idea are included in it, such as enlightenment, advertising, education, etc. (Bayer 1939: 17)

Entgegen dieser frühen Einsicht in die kommunikative Funktion der Ausstellungsgestaltung werden heute die Gestaltung einer Ausstellung und ihr Inhalt immer

15 Explizit beklagt Liebelt diese Tatsache und versucht, die Bedeutung der Architektur für das museumspädagogische Handeln in die Aufmerksamkeit zu rücken (Liebelt 1981). Leider hat dieser Sammelband wenig Auswirkungen auf die weitere Entwicklung der Forschung gehabt.

16 Auch unter den Etiketten „Museographie“, „Ausstellungsgestaltung“ oder „Szenographie“, s. Fülscher (2009), Baur (2016). 
wieder als Gegensätze aufgefass ${ }^{17}$ und Architektur und Ausstellungsdesign vorrangig unter dem Gesichtspunkt der Ästhetik, nicht dem der Kommunikation, betrachtet. ${ }^{18}$ Bücher zum Ausstellungsdesign sind oft nicht mehr als Dokumentationen ,guter` Ausstellungsbeispiele, in denen es meist um das künstlerische Gesamtkonzept und um die Beziehung zum ausgestellten (Gesamt-)Thema geht, während die Frage nach der Bedeutung, die durch ein einzelnes Designelement vermittelt wird oder nach dem semiotischen Zusammenspiel eines gestalterischen Elements mit einem bestimmten Exponat, einem bestimmten Text usw. nicht gestellt wird. In Handbüchern zur Ausstellungsproduktion (etwa Bertron/ Schwarz/Frey 2012, Lord/Piacente 2014) wird die Gestaltung von Ausstellungen vorrangig unter künstlerischen, praktischen, rechtlichen, konservatorischen oder rechtlichen Aspekten betrachtet, während es meist nur punktuelle Aussagen dazu gibt, wie welche Aspekte des Designs zur Vermittlung von Bedeutungen genutzt werden können. Dabei überwiegen allgemeine Aussagen zur grundsätzlichen Bedeutsamkeit von Elementen der Innenarchitektur und des Designs, während konkrete Analysen zum spezifischen kommunikativen Beitrag einzelner dieser Elemente oder zu der Frage, wie gestalterische und architektonische Elemente in einen Zusammenhang mit anderen im Raum präsenten Ausdrucksressourcen zu setzen sind, weitgehend fehlen.

$\mathrm{Zu}$ den wenigen Arbeiten, die systematisch der Bedeutung einzelner Elemente des ,Designcodes' nachgehen, zählen die schon mehrfach erwähnten Horta (1992 und 1991) sowie Jones (1999b und 1999a), die die Kommunikationsleistung der Architektur und Ausstellungsgestaltung unter dem Etikett des „design code“ untersuchen, Paul (2005), der etwa die Bedeutungspotenziale in der Architektur des Jüdischen Museums in Berlin untersucht, sowie Muttenthaler/Wonisch (2006), die von den genannten Autoren am stärksten die Tatsache berücksichtigen, dass Museumsarchitektur und -design in einer Wechselbeziehung zu ihrer multimodalen Umgebung stehen. Eng am architektonischen Detail arbeiten auch Naredi-Rainer (2004), der eine Reihe historischer und zeitgenössischer Museumsbauten auf die kommunikativen Implikationen ihrer Bauelemente und Raumprogramme hin untersucht, Felfe (2004) zur historischen Entwicklung der Blickführung durch Museumsarchitektur und -dispositive (wie Dioramen, „Sehmaschinen“), Parmentier (2007) zur Steuerung des Verhaltens durch die „dienende“ Museumsarchitektur und schließlich Stenglin (2009) und Ravelli

17 Vgl. Noschka-Roos (2001: 89-91), die Design und Architektur explizit aus dem „Informationskonzept“ einer Ausstellung ausschließt.

18 Vgl. Waidacher (2005: 142): „Nicht Semiotik ist der Schlüssel zur erfolgreichen Präsentation, sondern Ästhetik.“ 
(2006), die ihre Analyse der Bedeutungen der Ausstellungsarchitektur mit Hilfe des systemfunktionalistischen Beschreibungsinventars organisieren.

Da es also wenig Arbeiten aus dem Museumsbereich gibt, an denen die Frage anknüpfen kann, wie Elemente der Ausstellungsarchitektur und des Ausstellungsdesigns zur Konstruktion der Ausstellungsbedeutung beitragen können (betrachtet wieder vom Standpunkt der Rezeption aus), bietet es sich an, für die Untersuchung dieses Aspekts der Ausstellungskommunikation auf allgemeine Arbeiten zur Bedeutung von Architektur oder zu ihren kommunikativen Funktionen zurückzugreifen. Hierzu zählen beispielsweise

- Architekturtheorie (etwa Alexander et al. 1995 [1977], der „Muster“ in der Architektur als Lösungen wiederkehrender Probleme analysiert und sich damit als Anknüpfungspunkt für meine Sicht des Ausstellungsraums anbietet, oder Norberg-Schulz 1968, der der Analyse Begriffe zur Beschreibung von Raumrelationen zur Verfügung stellt);

- angewandte Architekturforschung wie z.B. der Ansatz der Space Syntax (ausgehend von Hillier 1996);

- Architektursemiotik (s. hier die Forschungsüberblicke von Dreyer 2003 und Nöth 1985a; in Trifonas 2015 findet man interessanterweise nur einen Artikel über Architekturzeichnungen, nicht aber über Semiotik der Architektur); und schließlich

- systemfunktionalistische Auseinandersetzungen mit Architektur (einen Überblick und zahlreiche Literaturangaben gibt Stenglin 2011).

Wenngleich der Forschungsüberblick dieses Abschnitts - den Traditionen des Felds folgend - nach den drei ,musealen Codes' Objekte, Texte und Design aufgebaut war, kann und wird sich die Analyse der Kommunikation durch die Ausstellung in Kapitel 3 nicht an dieser traditionellen Unterteilung orientieren. Denn die Ausstellungsbedeutung entsteht nicht, wie wir noch sehen werden, durch die separate Auswertung einzelner ,Codes‘, sondern im Gegenteil durch deren komplexe Verschränkung und gegenseitige Ausdeutung. Wie diese Verschränkung im Raum signalisiert wird, ist ein wichtiger Aspekt der Untersuchungen in Kapitel 3. Eine Analyse, die versucht, das Funktionieren der Ausstellungskommunikation zu rekonstruieren und dabei Code für Code vorgeht, würde die komplexen intercodalen Beziehungen, die ein Charakteristikum der Ausstellungskommunikation sind, künstlich unterbrechen und könnte so dem Untersuchungsgegenstand nicht gerecht werden.

Im restlichen Kapitel werde ich mich nun den Anknüpfungspunkten für die Untersuchung der Kommunikation durch die Ausstellung zuwenden, die sich aus der Linguistik, speziell aus der Textlinguistik, sowie aus einigen benachbarten 
Forschungsdisziplinen ergeben, etwa Semiotik und Kommunikationswissenschaft.

\subsection{Linguistische Anknüpfungspunkte}

Wie in Kapitel 1 ausgeführt wurde, ist die Kommunikation durch die Ausstellung durch die Multimodalität der zur Anwendung kommenden Ausdrucksressourcen, durch ihre spezifische Raumgebundenheit sowie ihre Ausrichtung auf die Vermittlung von Wissen gekennzeichnet. Diese Merkmale waren in den letzten Jahren Gegenstand intensiver Forschung. Im Folgenden werde ich zunächst die Grundzüge der systemfunktionalistischen und linguistischen Multimodalitätsforschung skizzieren (2.2.1) und daraufhin die ebenfalls stark im Kommen begriffene Forschung zur Räumlichkeit der Kommunikation und zur Ortsgebundenheit von Diskursen (2.2.2). Abschnitt 2.2.3 ist schließlich der Forschungen zur Wissenskommunikation gewidmet, speziell den Arbeiten, die sich für die lokale Konstruktion von Wissen interessieren.

\subsubsection{Multimodalität}

\subsubsection{Was ist ein Modus? Die systemfunktionalistische Multimodalitätsforschung}

Der zentrale Begriff der Multimodalitätsforschung ist der des mode bzw. des Modus. Die Debatte, wie dieser Begriff zu definieren ist, begleitet die Multimodalitätsforschung schon seit ihren Gründungstagen (aktuelle, ausführlich argumentierte Definitionsvorschläge finden sich in Wildfeuer/Bateman 2018 oder Stöckl 2016). Hier sollen einige ihrer Grundzüge dargestellt werden, die besonders relevant für das Verständnis der Kommunikation durch die Ausstellung sind.

Ein Modus besteht aus geregelten Zuordnungen von Ausdrucks- und Inhaltseinheiten - in den Worten von Kress und van Leeuwen: einer ,Grammatik‘ (Kress/van Leeuwen 1996: 1). Norris (2004: 12f., 158) definiert Modus als „a semiotic system with rules and regularities attached to it“ (mit Bezug auf Kress/ van Leeuwen 2001). Andere Autoren dagegen vermeiden das Wort System und sprechen stattdessen vorsichtiger von Zeichensets, Zeichenvorräten oder (wie in Kapitel 1 geschehen) von Ausdrucksressourcen. Diese Vorsicht ist eine Reaktion auf die Tatsache, dass in manchen Modi die Zeichen untereinander wesentlich lockerer verbunden sind, als das der strukturalistische Systembegriff vorsieht (Stöckl 2016: 10 fasst dies als unterschiedliche „Grade der Semiotisierung“). Auch hat die Multimodalitätsforschung Modi untersucht, deren Elementen sich nicht 
in jedem Einzelfall eine klar formulierbare, klar abgrenzbare und klar konventionalisierte Bedeutung zuordnen lässt. Man denke beispielsweise an die Beleuchtung als einen Modus der Kommunikation durch die Ausstellung. Ein Spot, der auf eine Steinplatte mit Versteinerungen gerichtet ist, hat zweifellos eine Funktion für die Ausstellungskommunikation: Er signalisiert, dass die Steinplatte als Exponat zu verstehen ist. Aber kann man sagen, dass der Spot im gleichen Sinne etwas bedeutet wie die Wörter der die Steinplatte begleitenden Objektkennung („Pterophyllum jaegeri BRONGNIART / Keuper / Steigerwald bei Bamberg“)?

Beobachtungen wie diese haben $\mathrm{zu}$ Versuchen geführt, Minimalanforderungen für Modi zu formulieren. Kress (2009) beispielsweise fordert, ein Modus müsse mindestens die drei Halliday’schen Metafunktionen „,ideational“, „interpersonal“ und „textual“ ausdrücken können; mit anderen Worten, er muss einen Sachverhalt repräsentieren können, eine Beziehung zwischen Kommunikationsbeteiligten stiften können, und strukturierte Botschaften ermöglichen. Diesen letzten Punkt betont Stöckl (2006: 21f.), der für die Botschaften eines Modus ein Mindestmaß an Komplexität fordert. Diese könne sich allerdings auch durch eine Verknüpfung zu anderen Modi ergeben, weshalb die Fähigkeit, eine solche intermodale Verknüpfung zu stiften, für Stöckl (ebd.) eine weitere Anforderung an einen Modus darstellt (vgl. auch das Konzept der „intermodal cohesion“ in van Leeuwen 2005).

In noch einer weiteren Hinsicht ist mit der Untersuchung der Modi eine Erweiterung des Bedeutungsbegriffs verbunden. Die systemfunktionalistische Beschäftigung mit Modi und dem Phänomen der Multimodalität (siehe z.B. Kress/ van Leeuwen 1996, Kress/van Leeuwen 2001, Norris 2004, O’Halloran 2004, Kress 2010, Bateson 2016, Tan/O’Halloran/Wignell 2020) ist Teil einer größeren Forschungsanstrengung, die bestrebt ist, den Zusammenhang von Zeichensystemen und ihrem Gebrauch durch die Zeichennutzer im Rahmen kommunikativer Praktiken zu modellieren (einen guten Überblick bieten Jewitt 2009 oder Norris 2013). Zeichen und Zeichensysteme werden aus dieser Perspektive von konkreten, sozial, kulturell und/oder historisch bestimmbaren Gruppen hervorgebracht, innerhalb derer sie verwendet werden und ihre Gültigkeit besitzen. Zeichen und Zeichensysteme sind flexibel: Sie werden an ihre spezifischen Verwendungskontexte und die in ihnen verfolgten Zwecksetzungen angepasst. Für die Bedeutung von Zeichen heißt das, dass sie nicht ein für allemal festgelegt ist, sondern immer offen für Spezifizierungen, Erweiterungen, Verschiebungen oder Umgewichtungen. Oder wie Stöckl (2006: 15) schreibt:

Die klassische Vorstellung fester Form-Inhalts-Koppelungen ist einem Konzept gewichen, nach dem eine Zeichenform ein Bedeutungspotenzial [...] eröffnet, das je nach Kommunikationssituation und -erfahrung [...] verschieden aktiviert werden kann. 
Bedeutung entsteht also erst, wenn Zeichenbenutzer die Zeichen im Rahmen ihrer kommunikativen Praktiken einsetzen (Kress/van Leeuwen 2001: 4). Die Verwendung des Begriffs Modus anstelle von Zeichensystem signalisiert diese spezielle Perspektive auf Signifikations- und Kommunikationsprozesse, die die aktive Rolle der Zeichenbenutzer bei der Herstellung von Bedeutung, die Verbindung von Zeichen und kommunikativen Zwecksetzungen und die Kontextabhängigkeit der Bedeutung in den Vordergrund rückt.

Dass Modi das Resultat von sozialen Konstruktionsprozessen sind, gilt nicht nur für die Bedeutung der einzelnen Zeichen. Auch die Frage, was zu einem Modus gehört, wird durch gesellschaftliche Definitionsprozesse beantwortet: „[A] mode is what a community takes to be a mode and demonstrates that in its practices“ (Kress 2009: 59).

Die Ausdrucksformen, die zu einem Modus gehören können, unterliegen nur sehr allgemeinen Beschränkungen, z.B. dass sie ein Set von Ausdrucksformen bilden sollen „with discernable regularity, consistency and shared assumptions about their meaning-potentials“ (2009: 59). Hinzu kommt, dass Modi nicht auf ein bestimmtes Medium beschränkt sind (Kress/van Leeuwen 2001: 23).

Das führt zu dem generellen Kategorisierungsproblem, nach welchen Kriterien bestimmt werden soll, welche Zeichen $\mathrm{zu}$ einem Modus gehören und wie der fragliche Modus bezeichnet werden soll. Auf die Kommunikation durch die Ausstellung bezogen: Gibt es beispielsweise einen umfassenden Modus Material, zu dem sowohl das Material der Vitrinen gehört wie das der Exponate? Oder ist Material einmal als Submodus des Modus Ausstellungsmöbel zu analysieren, ein andermal als Submodus des Modus Objekte? ${ }^{19}$ Muss man aus entfernbaren Elementen gebildete Durchlässe zwischen Ausstellungsräumen dem Modus Ausstellungsdesign zuordnen, vom Gebäude vorgegebene Durchlässe aber dem Modus Museumsarchitektur? Oder gehören sie $\mathrm{zu}$ einem einheitlichen Modus (und wie hieße der)?

Hier sind zwei grundsätzliche Antworten vorgeschlagen worden. Entweder beruft man sich auf Kategorisierungen der Zeichennutzer. Dies tut etwa Kress in dem oben schon angeführten Zitat ,a mode is what a community takes to be a mode and demonstrates that in its practices" (Kress 2009: 59). Im Einzelfall dürfte dieser Nachweis schwer zu führen sein, wie es auch unklar bleibt, welche Gruppe von Zeichennutzern diejenige ist, die für die Annahme eines Modus maßgeblich ist (s. dazu Bateson 2016: 39f.): die Ausstellungsmacher? die Verfasser von Handbüchern zur Ausstellungsgestaltung? die Besucher? Oder man entscheidet sich

19 Einen Vorschlag zur konsistenten Benennung unterschiedlich großer Einheiten in der Multimodalitätsforschung unterbreitet Stöckl (2004b: 14). 
wie Norris (2004) dafür, in Modi lediglich Untersuchungswerkzeuge zu sehen, die nur danach zu beurteilen sind, ob sie fruchtbare Einsichten in den jeweiligen Untersuchungsgegenstand erlauben. In diesem Sinne betont Norris, Modi seien „never a bounded or static unit, but always and only a heuristic unit“ (2004: 12). Statt sich zunächst an der hier dargestellten Definitions- und Kategorisierungsproblematik abarbeiten zu müssen, kann man als Analysierender mit dem Modus-Begriff eine Reihe von Phänomenen zusammenfassen, die man intuitiv als gleichgelagert wahrnimmt. Das erlaubt es, vorläufig von einem Modus Inneneinrichtung zu sprechen und diesen zu nutzen, um Beobachtungen in den Daten zu organisieren, ohne vorher definiert zu haben, wie jener von dem Modus Möbel abzugrenzen ist. Ob sich die Annahme halten lässt, dass Inneneinrichtung ein Modus ist, darüber entscheidet letzten Endes die Fruchtbarkeit der Einsichten, die ausgehend von dieser Untergliederung der Daten gewonnen werden können.

Die Frage, welchem Modus eine bestimmte kommunikative Erscheinungsform angehört, ist allerdings vor allem dann von Bedeutung, wenn die Analyse der multimodalen Kommunikation mit der Analyse je eines Modus beginnt, bevor dann in einem zweiten Schritt die Beziehung $\mathrm{zu}$ anderen Modi bestimmt wird. Diesen Weg gehe ich in der Untersuchung der Kommunikation durch die Ausstellung nicht. Stattdessen folgt die Analyse der Frage, wie bestimmte kommunikative Aufgaben im Ausstellungsraum bearbeitet werden (s.u. 3.2.6). Die Frage, welchen Modi die Phänomene angehören, die eine kommunikative Aufgabe bearbeiten, spielt dabei - wie sich zeigen wird - nur eine untergeordnete Rolle.

Überhaupt scheint mir die Leistung des Modus-Begriffs nicht darin zu bestehen, einen multimodalen Untersuchungsgegenstand in saubere ,Schnitte $z u$ zerteilen. Vielmehr eröffnet der Modus-Begriff den Blick für die kommunikative Leistung auch und gerade von Modi, denen wegen ihrer schwer zu fassenden Bedeutung oder ihrer losen internen Strukturierung bisher nicht die Aufmerksamkeit der am Vorbild Sprache geschulten linguistischen Forschung galt. Und der Modus-Begriff mit seiner Fokussierung auf die Wandelbarkeit von FormBedeutung-Relationen ermöglicht es, die aktive Leistung der Zeichennutzer bei der Konstruktion von Bedeutungen - etwa beim Gang durch die Ausstellung mitzuberücksichtigen.

\subsubsection{Die linguistische Multimodalitätsforschung}

Zwar setzt die linguistische Untersuchung multimodaler Kommunikation nicht erst mit der Prägung des Begriffs Multimodalität durch den Systemfunktiona- 
lismus ein, ${ }^{20}$ doch hat die Rezeption dieses Begriffs über Werke wie Kress/van Leeuwen (1996, 2001) zweifellos zu dem gegenwärtig zu beobachtenden Boom der linguistischen Multimodalitätsforschung maßgeblich beigetragen. In der Beschäftigung mit multimodaler Kommunikation begegnen sich unterschiedliche linguistische Subdisziplinen, vor allem die Stilistik, die Textlinguistik und die Medienlinguistik. ${ }^{21}$ Trotz unterschiedlichen disziplinären Zugängen ist hier ein enger Diskussionszusammenhang entstanden, in dem sich eine Reihe von Themen ausmachen lassen, die - in unterschiedlicher Gewichtung und Kombination - in vielen Arbeiten zu finden sind und eine Art gemeinsames Arbeitsprogramm ergeben.

Nachdem lange Zeit unter Textanalyse ausschließlich die Analyse des Wortlautes verstanden worden war, hat sich sowohl in der Stilistik (schon früh: Spillner 1982, Fix 1996) als auch in der Textlinguistik und -semiotik (Eckkrammer/ Held 2006b, Stöckl 2004a) die Einsicht durchgesetzt, dass zur Textanalyse auch die Analyse nicht-sprachlicher Ausdrucksressourcen gehört. So schreiben Ulla Fix und Hans Wellmann in der Einführung zu ihrem die Breite der Text-Bild-Forschung illustrierenden Sammelband (Fix/Wellmann 2000a):

Läßt man sich darauf ein, Bilder als symbolische Zeichenkomplexe in sprachwissenschaftliche Untersuchungen einzubeziehen, stellt sich auch die Frage nach einem geeigneten Zeichen- und Textbegriff. Die sprachwissenschaftliche Begrifflichkeit ist daraufhin $\mathrm{zu}$ überprüfen, inwieweit sie es erlaubt, Sprachtexte und Bildtexte in einem Zugriff, mit darauf abgestimmten Kategorien zu beschreiben und zu erklären. (Fix/Wellmann 2000b: xiii)

Ein Schwerpunkt der linguistischen Multimodalitätsforschung ist daher eine Neubestimmung des Begriffs Text, eine breit geführte Diskussion, die beispielsweise in den Wettbewerbsbeiträgen ihren Niederschlag gefunden hat, die in Fix (2002) dokumentiert sind. Mit der Abkehr vom „logozentrische[n] Blick“ (Eckkrammer/Held 2006b: 1), der die Sprachwissenschaft traditionell geprägt hat, erwächst ein Interesse an all dem, was neben Sprache die Interpretation von Texten beeinflussen kann. Der Blick der Forschung richtet sich auf ihr konkretes Vorkommen in Lektüresituationen, ein Aspekt, den Fix (2008) mit dem Begriff

20 Älter als der Begriff Multimodalität ist etwa die Forschung zum Bild-Text-Verhältnis (s. z.B. Bentele/Hess-Lüttich 1985, Hupka 1989 zu Enzyklopädien, Krafft 1978 zur textlinguistischen Analyse von Comics).

21 Andere sprachorientierte Disziplinen, die sich mit Multimodalität befassen bzw. schon früh befasst haben, sind die Rhetorik (etwa in Gestalt der Versuche, rhetorische Konzepte für die Filmanalyse fruchtbar zu machen, Knilli et al. 1971), überhaupt die Film- und Fernsehforschung (Straßner 1985, Kanzogs Versuch der Begründung einer „Filmphilologie“, Kanzog/Burghardt 1991). 
der „Lokalität“ oder Sandig (2000: 18) mit dem der „Situationalität“ zu fassen versuchen und den Hausendorf/Kesselheim (2008) zum Ausgangspunkt ihrer Textanalysen machen. ${ }^{22}$

Auch entsteht ein Interesse an der Materialität von Texten, also an der Form und an dem Material des Textträgers, an der verwendeten Drucktechnik, der Gestaltung der Schriftzeichen - kurz: an all den Sinneseindrücken, die der Text dem Leser im Moment der Lektüre zur Verfügung stellt. Zwei Aspekte der Materialität von Texten haben besondere Aufmerksamkeit geweckt: zum einen, dass Schrift als Phänomen in der Fläche angesehen werden kann (Gross 1994), zum anderen an dem „Bedeutungsüberschuss“ des sprachlichen Zeichens, der durch seine Materialität entsteht (Bucher 2007 zur „Resemiotisierung der Form“).

Die Beschäftigung mit den Neuen Medien und ihren Kommunikationsformen, in denen Multimodalität und Multimedialität zum Normalfall geworden ist (s. etwa Wagner 2006, 2011; Dürscheid/Frick 2016), lässt ein allgemeines sprachund medientheoretisches Interesse an Medienübergängen und -kombinationen sowie den Strukturen und Leistungen einzelner Medien entstehen. ${ }^{23}$ Unter Etiketten wie dem der Medialität (Fix 2008) werden die speziellen Bedingungen der technischen Speicher- und Übertragungsmedien in die Analyse von Texten einbezogen und Konzepte wie das der „Transkription“ bzw. „Transkriptivität“ (Jäger, 2002, Jäger/Stanitzek 2002, Holly 2006) versuchen, den besonderen Bedingungen und Beschränkungen des in den einzelnen Medien Ausdrückbaren und dem Beitrag des Mediums zur Textbedeutung auf die Spur zu kommen (s. a. Krämer 1998). ${ }^{24}$

Mit der Untersuchung der Neuen Medien gerät auch die Tatsache in den Blick, dass viele Texte für eine nicht-lineare Rezeption ausgelegt sind, eine Entdeckung, die sich in Begriffen wie „Sehfläche“ (Gross 1994, von Schmitz 2004: 112 auf die Beschreibung von Texten in Neuen Medien angewandt) oder „PuzzleText“ (Püschel 1997) widerspiegelt. Dies führt auch zu einer Neubewertung der

22 Heinemann/Viehweger (1991: 157) bestreiten noch explizit die Bedeutung der Lektüresituation (bei ihnen „LOC“) für die Textanalyse, weil „mit dem Wechsel von LOC in der Regel nur eine Veränderung der Textinhalte, nicht aber auch intentionale, strategische oder Strukturwandlungen verbunden“ seien.

23 Arbeiten zum Thema Neue Medien deuten die beobachteten Phänomene oft als Beleg für größere historische Entwicklungen. Ein Beispiel dafür ist Schmitz (2007), für den die Kommunikationsgeschichte „im Großen und Ganzen [...] von der Textlinie über die Sehfläche zum virtuell dreidimensionalen Informationsraum“ verläuft“ (Schmitz 2007: 108).

24 Wenn die Beschäftigung mit der Medialität auch durch die Untersuchung der Neuen Medien unzweifelhaft einen Aufschwung genommen hat, sind die medialen Bedingungen der Kommunikation schon früher zum Thema geworden: s. etwa Gumbrecht/Pfeiffer (1988). 
Rolle des Rezipienten, der zunehmend als aktiver Konstrukteur von Bedeutungen wahrgenommen wird, der durch seine Entscheidungen erst den Text konstruiert (Schumacher 2009, Wienen 2011; diese Haltung wird auch hier vertreten, s.u. 3.2.1).

Schließlich führt die Untersuchung von Modi wie Typografie oder Layout (Wehde 2000, Stöckl 2004c, Spitzmüller 2006, 2016; Roth/Spitzmüller 2007, Antos/Spitzmüller 2007, die eher ,schwache Codes darstellen, zu einer erweiterten Vorstellung dessen, was Bedeutung ist. Hier berühren sich die text- und medienlinguistische und die oben vorgestellte systemfunktionalistische Multimodalitätsforschung. Gefordert wird, an die Stelle von repräsentationalen Vorstellungen vom Zeichenkörper-Zeicheninhalt-Verhältnis, die von einer ein für allemal fixierten, dem Zeichen quasi innewohnenden Bedeutung ausgehen, dynamische Bedeutungskonzepte zu stellen, die am Zeichengebrauch in der Kommunikation orientiert sind und die Bedeutungen als Resultat von Aushandlungsprozessen ansehen, in die die aktuelle Kommunikationsziele der Beteiligten eingehen.

\subsubsection{Eigenschaften von Modi und intermodale Beziehungen}

Systemfunktionalistische und linguistische Untersuchungen zur Multimodalität teilen sich eine Reihe von gemeinsamen Forschungsinteressen. Dazu gehört insbesondere die Frage, wie sich die spezifischen Ausdruckmöglichkeiten eines Modus erfassen lassen und wie intermodale Beziehungen $\mathrm{zu}$ beschreiben und $\mathrm{zu}$ klassifizieren sind.

Ein häufig beschrittener Weg, die grundlegenden Eigenschaften von Modi herauszuarbeiten, besteht darin, zwei Modi kontrastiv zu beschreiben und die identifizierten Unterschiede zu generalisieren. Diesen Weg haben zahlreiche Autoren beschritten, und zwar insbesondere bei der Beschreibung der Unterschiede von Objekt- und Sprachzeichen (zum Überblick: Nöth 2000b, s.o. 2.1.2), von Schrift und Bild (Worth 1981, Nöth, 2000a, 2016; Stöckl 2004a, 2016, Schmitz 2007, Machin 2007: 171-176) sowie von Gestik und Sprache (McNeill 1992: 19-23, 38f.).

Die von diesen Autoren beschriebenen Unterschiede und Klassifikationsvorschläge lassen sich zu folgenden Vergleichsdimensionen zusammenfassen:

- die Eigenschaften des Systems, wozu etwa die Existenz von Wohlgeformtheitsbedingungen, die gesellschaftliche Verbreitung des Codes, Segmentierbarkeit und Kompositionalität gehören (vgl. besonders McNeill 1992: 19-23);

- die Materialität der Zeichenkörper und ihre Folgen (z.B. die Linearität der gesprochenen Sprache, die Dreidimensionalität von Objekten usw.) - hierzu gehören Beobachtungen wie die von Schmitz (2007), der dem Modus „Tex- 
tualität“ die Eigenschaft zuschreibt „eher arbiträr-symbolische Zeichen“ zu besitzen (2007: 99);

- Semantik, also die Frage der Bedeutungen, die durch den Modus ausgedrückt werden können (bei Stöckl 2016: 15: „semantisches Potenzial“, mit Merkmalen wie vage vs. präzise, zuverlässig vs. unzuverlässig usw.); ausführlich Nöth 2016: 202-210

- Syntaktik - wenn Schmitz (2007: 99) von einer ,grammatisch-hierarchische[n] Organisation für sukzessive Bottom-up-Wahrnehmung“ spricht;

- Pragmatik: die kommunikativen Handlungen und Funktionen, die das Zeichensystem ermöglicht (Schmitz 2008, Nöth 2004: 20f., Stöckl 2016: 15-18, Nöth 2016: 196-202).

Diese Dimensionen sind meines Erachtens dazu geeignet, die charakteristischen Eigenschaften verschiedener Modi systematisch einander gegenüberzustellen und damit dem auf die Spur zu kommen, was Kress die „semiotic logic“ eines Modus oder seine „affordances“ nennt (Kress 2009: 56, s.a. Jewitt 2009: 24).

Viele Arbeiten gibt es dazu, welche Beziehungen verschiedene Modi in einem multimodalen Ensemble ${ }^{25}$ eingehen können. Häufig konzentrieren sich diese Arbeiten auf je eine Modus-Paarung, etwa das Verhältnis von Bild und Schrift in Printmedien (etwa Stöckl 2004a, Stegu 2000), in Fernsehnachrichten (Straßner 2002, Luginbühl 2011) oder in Apps und dem Internet (Symreck 2019, Dancygier/ Vandelanotte 2017, Siever 2015). ${ }^{26}$ Aus der Vielzahl der Untersuchungen lässt sich erneut eine kleinere Anzahl allgemeiner Dimensionen gewinnen, auf denen die intermodalen Beziehungen in einem multimodalen Ensemble beschrieben werden können:

- die Dimension der Semantik betreffen Vorschläge wie die „pauschalisierten Konstellationen“ in Stöckl (2006: 28) mit den Kategorien ,additiv“, „,komplementär“, „hierarchisch“, „divergent“ und „konfliktiv“; hierher gehören aber auch rhetorisch inspirierte Kategorien wie die der Metonymie oder Metapher (s. Stöckl 2004a, zum Film: Knilli et al. 1971);

25 Darüber, wie man die multimodalen Produkte nennen soll, herrscht Uneinigkeit: Stöckl (2016) verwendet „multimodale Texte“, „multimodale Kommunikate» oder „multimodale Artefakte“, Sandig (2000: 3) „Konglomerate“, „Text-Bild-Konglomerate“ oder „Sprache-Bild-Texte“ (Sandig 2000: 3). In dieser Arbeit spreche ich abwechselnd von „Ensembles“, „Arrangements“ oder „Kombinationen“.

26 Arbeiten, die nicht primär an der Klassifizierung intermodaler Verhältnisse interessiert sind, haben häufig eine größere Zahl von Modi im Blick: siehe etwa Girnth/Michel (2007) oder Luginbühl (2007). 
- die formale Dimension: Hier schlägt Nöth (2000a) vor zu untersuchen, ob es beispielsweise ein Nebeneinander von Bild und Text gibt oder ob beide ineinander verschränkt sind usw.;

- eine Dimension der Pragmatik, auf der nach den kommunikativen Funktionen gefragt wird, die dadurch ausgeübt werden, dass zwei Modi in Beziehung treten (z. B. die Funktion des Benennens, s. ebd.); und schließlich

- eine Dimension der Relevanz, in der die Frage beantwortet wird, ob einer der beteiligten Modi die Vorherrschaft über den anderen hat (hierauf zielt beispielsweise Nöths Kategorie der „Richtung der Verhältnisse“, Nöth 2000a). ${ }^{27}$

In frühen Arbeiten zur Bestimmung intermodaler Beziehungen war die Klassifizierung der Beziehungen eine dominante Orientierung. Die Analyse eines gegebenen multimodalen Ensembles zielte also im Wesentlichen darauf ab, die Beziehung der beteiligten Modi mit einem einzigen Begriff zu beschreiben. Die implizite Vorstellung war dabei die, dass Bild und Text je eine globale Aussage beinhalten, deren Bedeutungsgehalt vom Analysierenden in einem ersten Schritt bestimmt wird. Diese globalen Aussagen können dann miteinander verglichen und in Beziehung gesetzt werden, woraufhin die festgestellte Beziehung einer allgemeinen Kategorie zugeordnet wird - eine Vorstellung, die noch der Typologie von „Visualisierungs-Praktiken“ in Schmitz (2018: 259f.) zugrundeliegt.

Diese Vorstellung wird aber der Tatsache nicht gerecht, dass die Modi der untersuchten multimodalen Ensembles in aller Regel komplexe Botschaften bilden, die gleichzeitig eine Vielzahl unterschiedlicher Beziehungen auf zahlreichen Ebenen stiften können. Analysiert man beispielsweise das Verhältnis von einem Zeitungsartikel zu dem ihn begleitenden Bild, dann reicht es sicher nicht aus, die globale Aussage des Artikels (wie auch immer man zu dieser gelangen mag) mit der globalen Aussage des Bilds zu vergleichen. Um das Zusammenspiel von Bild und Text zu verstehen, muss man sicherlich auch einzelne Wörter oder Redewendungen im Text heranziehen, vielleicht die Bildunterschrift usw. und auf Seiten des Bildes auch einzelne Bildbestandteile wie abgebildete Personen oder erkennbare Situationstypen usw.

In der jüngeren Multimodalitätsforschung ist die Orientierung an der Klassifizierung globaler Beziehungen zugunsten eines Interesses an den vielfältigen, oft kleinräumigeren Beziehungen zwischen Zeichen(gruppen) aus verschiedenen Modi zurückgetreten. In diese Richtung geht etwa Stöckls Vorschlag, die kogni-

27 Hoffmann (2004: 359) bezieht in seine Analysen zusätzlich eine soziale Ebene ein. Seine Beispiele belegen aber sozialsymbolische Funktionen, die nicht durch den Bild-TextZusammenhang gestiftet werden, sondern durch die Bilder allein. 
tiven Grundlagen der „,intermodale[n] Kohäsivität“ (Stöckl 2006: 27) in den Blick zu nehmen. Stöckl verweist auf Assoziationen, Synästhesien, metaphorisch-metonymische Übertragungen, paradigmatische Sinnbezüge (Antonymie, Hyperonymie usw.) sowie logische Relationen wie Kausalität oder Finalität. Damit lenkt Stöckl den Blick auf die Frage, wie Rezipienten im Detail semantische Beziehungen zwischen Bild und Text herstellen und welche Indizien in Bild und Text sie dabei jeweils ausnützen können. Das gilt ähnlich auch für die Bestimmung der Beziehungen auf formaler oder pragmatischer Ebene. In Stöckl (2016) geht der Autor noch einen Schritt weiter hin zu einem Mehrebenenmodell der empirischen Beschreibung multimodaler Textensembles (2016: 22-25). Allerdings hält er fest:

Vor allem die Integration von Zeichenmodalitäten in multimodalen Gesamttexten ist m. E. noch unzureichend beschrieben, so dass die Verknüpfungsmuster von ,modes' und die Prinzipien der intersemiotischen Sinnstiftung einen weitestgehend blinden Fleck markieren.

Inspirierend ist in dieser Hinsicht auch Blunden (2020), die feinkörnig nachweist, wie über Übereinstimmungen und Kontaste zwischen dem verbal und dem bildlich Ausgedrückten (,vergence“) punktuelle „verbal vectors“ entstehen, die den Blick des Besuchers vom Text auf bestimmte Bereiche des Bilds lenken (s.u., 3.5).

Die Untersuchung der Kommunikation durch die Ausstellung knüpft an solche Ansätze an, die im Detail untersuchen, wie die Rezipienten eines multimodalen Ensembles semantische, formale und pragmatische Beziehungen zwischen Zeichen aus unterschiedlichen Modi herstellen und welche Elemente des multimodalen Ensembles ihnen die Herstellung dieser Beziehungen nahelegen. Dabei sollte die Analyse sowohl Beziehungen zwischen großen monomodalen Einheiten wie auch punktuelle Beziehungen zwischen einzelnen Zeichen aus je einem Modus (z.B. Exponat und Objektkennung!) erfassen können.

\subsubsection{Raumgebundenheit der Kommunikation}

Während der „spatial turn“ in anderen Geisteswissenschaften schon vor einiger Zeit vollzogen worden ist und damit die gesellschaftliche Herstellung des Raums und die Relevanz des Raums für unser Handeln und unsere Konstruktion von Sinn in den Blick gerückt ist (vgl. Döring/Thielmann 2008), hat sich in der Linguistik erst in jüngerer Zeit ein spezifisches Interesse am Raum und seiner Rolle in der Kommunikation ausgebildet. Der folgende Abschnitt soll aufzeigen, was die Linguistik zur Erforschung der Rolle des Raums in der Kommunikation durch die Ausstellung beitragen kann. 


\subsubsection{Räumlichkeit von Texten, Raum im Text, textuelle Bedeutungseffekte des Raums}

Schon die Beschäftigung mit der Materialität und Medialität von Sprache (s.o.) hat zu der Einsicht geführt, dass Raum eine relevante Kategorie für die Untersuchung sprachlicher Produkte sein kann. Gross stellt in ihrer Abhandlung zu Prozessen der Rezeption von Schrift und Bild (Gross 1994) die Räumlichkeit als ein bis dahin wenig beachtetes Merkmal der Schrift heraus. Schrifttexte seien keineswegs linear, sondern erstreckten sich über eine zweidimensionale Fläche. Ebenso wenig erfolge die Rezeption von Schrifttexten linear, sondern geschehe in einer Reihe von Sakkaden und Fixationen auf der „Lesefläche“ (1994: 61), besonders bei der Wahrnehmung der Gesamtgestalt und der Gliederungseinheiten des Schrifttexts - empirisch nachgewiesen durch Eye-Tracking-Studien (s. Clifton Jr. et al. 2016). Wenn Raum hier auch als strukturelle Eigenschaft von Texten in das Blickfeld der Untersuchung gerät, endet die Analyse doch noch ,am Rand des Papiers'. Die Frage, wie die „Lesefläche“ der Schrift oder des Bilds eingebunden ist in die räumliche Umwelt, in der die Rezeption stattfindet, liegt für Gross außerhalb des Untersuchungsinteresses.

Ähnliches gilt für textlinguistische und textsemiotische Arbeiten, die die Verbalisierung des Raums in Texten untersuchen. Diese bauen in der Regel auf Forschungen auf, die das Verhältnis von Raumkognition und Sprache oder aber die sprachstrukturellen Voraussetzungen des Ausdrucks von Raum (Raumkategorien in Lexik und Grammatik, Raumdeixis und -referenz) untersucht haben. ${ }^{28}$ Ein Beispiel hierfür ist Wenz (1997), die einen London-Reiseführer und einen London-Roman analysiert, um zu einer „Beschreibung der semiotischen Beziehungen zwischen Raumkognition und ihrer textuellen Repräsentationen“ (1997: 17f.) zu gelangen. Hinter den „semiotischen Beziehungen“ verbirgt sich im Wesentlichen die Einteilung der sprachlichen Bezugnahmen auf Raum entsprechend der bekannten Peirce'schen Zeichenklassifikation und die „textuellen Repräsentationen“ werden auf das „Linearisierungsproblem“ (1997: 57) reduziert, also die Frage, wie die dreidimensionalen Tatsachen des Raumes in die lineare Abfolge der Sprachzeichen im Text transformiert werden. ${ }^{29}$ Das Linearisierungsproblem steht auch im Mittelpunkt von Schubert (2009) und Schmauks (2002: besonders

28 S. etwa Sweetser/Gaby (2017), Tenbrink (2017).

29 Der Linearisierungs-Perspektive liegt eine doppelte Idealisierung zugrunde: eine zeitlose Raumwahrnehmung, in der alle räumlichen Entitäten und Relationen gleichzeitig präsent sind, und eine Textrezeption, die ausschließlich linear verläuft. - Frühe linguistische Arbeiten zur Linearisierungsproblematik sind Ullmer-Ehrich (1979) oder die Beiträge zu Wegbeschreibungen in Jarvella/Klein (1982: Part 2, 161-268). 
89-93). Schmaucks untersucht, wie ein Wanderführer den Aufbau eines mentalen Modells der beschriebenen Gegend unterstützt und so zur räumlichen Orientierung von Wanderern beiträgt. Obwohl der Nutzen von Wanderführern in hohem Maße davon abhängt, dass es den Lesern im Moment der Rezeption gelingt, eine Verbindung zwischen dem Text und ihrer aktuellen räumlichen Umwelt herzustellen, geht die Autorin diesem Aspekt des Text-Raum-Zusammenhangs nicht systematisch auf den Grund. In den aufgeführten Arbeiten geht es zwar um Texte und Raum, aber nicht darum, wie Texte in die sie umgebende räumliche Umwelt eingebettet sind und wie sie diese (mit)konstituieren, sondern wie in Texten räumliche Sachverhalte und Beziehungen bzw. deren mentale Repräsentationen zum Ausdruck kommen.

Die Stilistik hat sich schon früh mit den Bedeutungseffekten beschäftigt, die sich aus dem Raumbezug von Texten ergeben (s. etwa Spillner 1982). Zu den Autoren, die dieses Thema ausführlicher behandelt haben gehören Ulla Fix und Barbara Sandig.

Fix fordert, die Einbettung von Texten in ihre räumliche Umgebung in der textstilistischen Analyse mitzuberücksichtigen (Fix 2008). Diesen Aspekt behandelt sie unter dem Schlagwort Lokalität:

Lokalität bezeichnet den Ort der Publikation. Der institutionalisierte - kulturell verfestigte - Ort, an dem eine Mitteilung publiziert wird, hat auch eine - kulturell verfestigte - Bedeutung, die dem Rezipienten ebenso wie Medium und Materialität etwas zu verstehen gibt. (ebd.: 345$)^{30}$

Die Verbindung von Texten $\mathrm{zu}$ diesen kulturell definierten Situationstypen erlaubt unterschiedliche Arten von Bedeutungseffekten, wobei Fix zwischen einem „usuelle[n]“ und einem „okkasionelle[n] Gebrauch“ von Lokalitäten unterscheidet: Im „usuelle[n] Gebrauch“ stellt die Lokalität die grundlegende Bedingung für das Vorkommen des Texts dar, im „okkasionelle[n] Gebrauch“ kann sie die Textfunktion verstärken oder der Auslöser für die Zuordnung des Texts zu einer bestimmten Textsorte sein (ebd.: 349f.).

Barbara Sandig behandelt in ihrer Textstilistik des Deutschen die Einbettung von Texten in den sie umgebenden Raum als eine der „Merkmalsausprägungen“, die die spezifische Textualität eines Texts ausmachen (2006: Kap. 5), gleichrangig neben Merkmalen wie „Funktion“, „Thema“, „Kohäsion“ und „Kohärenz“, die

30 Domke (2010a: 89) kritisiert, der Begriff Lokalität erlaube nicht, zwischen ortsgebundenen und an Gegenstände gebundenen Texten zu unterscheiden. Für Fix seien ein Buchdeckel und eine Uferbefestigung gleichermaßen ,Orte'. Das gilt übrigens auch für Auer (2010), der wiederholt von „orts- und dingfesten“ Zeichen spricht, ohne diese beiden Aspekte zu unterscheiden. 
von der Textlinguistik bisher als grundlegende Textualitätsmerkmale diskutiert worden sind. Für Sandig tritt Raum nicht mehr nur als Teil des „Kontexts“ additiv zur ,eigentlichen` Textanalyse hinzu, um ansonsten schwer handhabbare Bedeutungseffekte zu erklären, sondern ist grundlegender Bestandteil der stilistischen Textanalyse. Für das hier verfolgte Interesse finden sich nützliche Beobachtungen unter dem Schlagwort „Ortspragmatik“ oder „pragmatischer Ort“.

\subsubsection{Discourses in Place}

Ein breit rezipiertes Werk, das für die Erforschung des Vorkommens von Texten in der räumlichen Umwelt (speziell: im öffentlichen Raum) höchst folgenreich war, ist Scollon/Scollon (2003). Dort wird der in M.A.K. Hallidays Sozialsemiotik verankerte Ansatz der „geosemiotics“ entwickelt. Einen guten Überblick über die Grundlinien der Geosemiotik gibt Al Zydaly (2014).

Die Untersuchung der Einbettung von Texten in die ,materielle Welt' hat nicht primär zum Ziel, das Verständnis des Texts zu erweitern (also etwa zu verstehen, mit welchen sprachlichen und nicht-sprachlichen Mitteln ein Text mit seiner räumlichen Umwelt in Beziehung treten kann oder wie der Ort der Lektüre eine bestimmte Textsorteninterpretation fördert oder blockiert). Vielmehr geht es den Autoren vorrangig um die Frage, wie das Sprachsystem „indexes the other semiotic systems in the world around the language“ (2003: 5), also gesellschaftliche oder politische Machtbeziehungen, die Positionierung in sozialen Kategorien wie Nationalität oder ethnischer Zugehörigkeit, die Signalisierung von Werteorientierungen usw. (2003: 12). Auch wenn der Ausdruck sozialer Bedeutungen, die mit der Verankerung von Texten im Raum ausgedrückt werden können, nicht im Vordergrund meiner Untersuchung steht, werden in diesem Buch dennoch Analysekategorien entwickelt, die für die Analyse der Ausstellungskommunikation nützliche Anknüpfungspunkte bieten. Konkret handelt es sich etwa um

- das Konzept der „inscription“ (2003: 129), mit dem die Materialität von Zeichen und Zeichenträgern gefasst wird, inklusive ihrer Zeitlichkeit, die mit dem Begriff des „layering“ gefasst wird (etwa wenn auf ein Theaterplakat ein Schild „heute“ geklebt wird, 2003: 137);

- die Unterscheidung zwischen ,indexikalischen“ und ,symbolischen“ Funktionen von Zeichen (bei ersterer entsteht die Bedeutung durch die Platzierung oder die Materialität des Zeichens, bei letzerer dadurch, dass der Zeichenkörper auf etwas Abwesendes verweist, 2003: 133);

- das Konzept des „emplacement“, das die Art und Weise der Verbindung zur Umwelt betrifft (2003: Kap. 8): Zeichen können als „decontextualized“, „situated“ oder „transgressive“ klassifiziert werden; ein ,situiertes“ Zeichen „is shaped by and shapes the material world in which it is placed (2003: 22), 
ein ,dekontextualisiertes' dagegen signalisiert seine Unabhängigkeit vom Kontext, und ,transgressive` Zeichen verstoßen gegen Platzierungskonventionen oder -vorschriften;

- und schließlich die Betonung der Tatsache, dass Zeichen immer im Kontext anderer Zeichen zu sehen sind, mit denen sie „semiotic aggregates“ bilden (2003: 23, ausführlich: Kap. 9).

Analysiert werden vor allen Dingen Schilder und kleine Texte (wie private Annoncen) im öffentlichen Raum. Die Analyse der sprachlichen Komponente der Schilder und Texte beschränkt sich allerdings im Wesentlichen auf die Wahl der Sprache(n) und, bei mehrsprachigen Texten, auf die Reihenfolge der beteiligten Sprachen. Das erklären die Autoren damit, dass ,Textsysteme' schon mit großer Ausführlichkeit untersucht worden seien (2003: 7). Doch hat diese an sich gerechtfertigte Entscheidung Folgen: Zum einen lässt die geringe Detailtiefe, mit der die Texte untersucht werden, den Eindruck entstehen, es käme bei der Analyse der discourses in place nur auf die globale Charakterisierung der Beziehung von Text und Raum an, ohne dass man den Mikrophänomenen nachgehen müsste, die diese Verbindung erzeugen (z. B. Raumlexik, Deiktika, grafische Verknüpfungsmittel usw.). Zum anderen führt die weitgehende Ausblendung der Analyse der sprachlichen Formen dazu, dass die Analyse der Einbettung von Texten in die ,materielle Welt' als etwas erscheint, das unabhängig (und vielleicht sogar: anstelle) von einer Analyse der sprachlichen Formen zu leisten wäre.

Das gilt auch für den der Geosemiotik nahestehenden Ansatz der linguistic landscapes (einleitend Gorter 2006; Backhaus 2007, Peck/Stroud/Williams 2019). Dieser Ansatz untersucht insbesondere das Vorkommen von Zeichen in Großstädten in mehrsprachigen Gesellschaften (s. etwa Laskurain-Ibarluzea 2020, Blommaert/Maly 2016, Schmitz/Ziegler 2016). Im Mittelpunkt des Interesses steht die Frage, wie Schrift im öffentlichen Raum Territorien markiert, Machtverhältnisse zwischen verschiedenen Gruppen abbildet und so einen spezifischen Raum konstituiert. Im Vordergrund der Analysen steht, dem Fokus auf Mehrsprachigkeit entsprechend, der Aspekt der Sprachwahl und seinen Bedeutungen. Neuere Arbeiten weiten das Interesse auf Zeichen im öffentlichen Raum und den daran anknüpfenden Diskursen aus (z.B. Schmitz/Ziegler 2016, Scarvaglieri/Luginbühl 2020, Seargeant/Giaxoglou 2020).

Während Arbeiten aus der Geosemiotik und dem Linguistic-LandscapeAnsatz vorrangig an den sozialen Bedeutungen interessiert sind, die mit dem Vorkommen von Zeichen im öffentlichen Raum verbunden sind, geht es anderen Arbeiten, wie beispielsweise Auer (2010) um die grundsätzliche Klärung der Frage, wie „öffentliche, orts- und dingfeste Zeichen überhaupt Raum konstituieren“ (2010: 271). Dazu beschreibt Auer die möglichen Relationen zwischen 
Zeichen(ensembles) und Raum präziser und unter Berücksichtigung konkreter sprachlichen Formen. ${ }^{31}$ Bei den ,situierten‘ Zeichen (die bei Auer „indexikalisch“ genannt werden, 2010: 278f.) unterscheidet er beispielsweise, ob eine „Kontiguität von Zeichen und intendiertem Referenten“ vorliegt (illustriert an einer Aufschrift auf einem Objekt, die den Besitzer des derart beschrifteten Objekts angibt) oder ob auf etwas Entferntes verwiesen wird (wie im Fall eines Wegweisers) und ob die Funktion der Schrift einen Ort als „Thema der auf dem Zeichen verbalisierten rhematischen Information“ ausweist oder ob zur Interpretation des Zeichens zunächst ein gewisses Wissen aktiviert werden muss (illustriert an dem Schild „Kasse im EG“ in einem Parkhaus). Weiterhin differenziert Auer das Scollon'sche Konzept der „semiotic aggregates“ und gelangt so zu einer differenzierteren Beschreibung der Zusammenhänge, die zwischen verschiedenen ortsgebundenen Zeichen bestehen können. Auer (2010: 286f.) unterscheidet

- ein „Ensemble“, das dadurch definiert ist, dass die dazugehörigen Elemente mit einem Blick wahrzunehmen sind,

- einen „Zeichendiskurs“ aus Elementen, deren Gruppierung auf einer „formale[n] und funktionale[n] Ähnlichkeit“ beruht, und

- eine „Schichtung“ (d.i. das Scollon’sche „layering“): eine Kombination von Zeichen, die es erlaubt, auf eine zeitliche Abfolge der Anbringung zu schließen.

Während es in den bisher dargestellten Arbeiten um Aspekte von Semantik und Syntaktik der Zeichen im öffentlichen Raum geht, widmet sich Auer, meines Wissens als Erster, dem Aspekt der Funktionen von Zeichen im öffentlichen Raum. Die von ihm identifizierten Funktionen umfassen:

1) Benennen und Charakterisieren. 2) Zugehörigkeit markieren, 3) Gebrauchsweisen vorschlagen oder verbieten, 4) Wege weisen sowie 5) Ermahnen und Gedenken (2010: 290).

$\mathrm{Zu}$ alternativen Listen von Funktionskategorien gelangen Cook (2013: 53-67: „locating and attracting“, „informing“, „controlling movement and behaviour“ sowie die problematische Kategorie „,service signs“) oder Schmitz/Ziegler (2016). Letztere unterscheiden zwischen „Information“, „Regulierung“, „Werbung“, „Selbstinszenierung des Urhebers“ und „Meinungskundgabe“ (2016: 479). Besonders interessant ist aber ihre funktionale Beschreibung der Relationen zwischen

31 Anders als Scollon/Scollon (2003) geht Auer auf die sprachlichen Formen ein, durch die die Verbindung zum Raum hergestellt wird, etwa das Vorhandensein lokaler Deiktika (Auer 2010: 279). 
Zeichen im öffentlichen Raum, die aufeinander Bezug nehmen. Hier unterscheiden sie „Anerkennung“, „Kritik“, „Konkurrenz“ und die bloße „Präsenz“ (2016: 483). Durch diese Beschäftigung mit den Textfunktionen nähern diese Autoren die Analyse der Raumgebundenheit von Texten an die textlinguistische Tradition der Textanalyse an, für die die Ermittlung der Textfunktion traditionell ein wichtiger Aspekt ist (s. 3.7).

Der grundlegenden Rolle der Raumgebundenheit von Texten für die Kommunikation geht auch Domke nach (s. Domke 2010a, 2010b, 2013). Die Autorin untersucht

Kommunikationsformen [...], die über ihre Funktion [...] an (geographisch) bestimmbare Orte und Räume der Interaktion bzw. Rezeption gebunden sind (Domke 2010a: 87).

Konkret handelt es sich um Texte, die im öffentlichen Raum zu sehen sind, etwa in Bahnhöfen oder Flughäfen. Diese beschreibt sie als einen Fall von „Mesokommunikation“, einer Form von Kommunikation zwischen Massenmedium und Face-to-face-Kommunikation, deren konstitutive Merkmale „die inhaltliche und/ oder medial-materiale Gebundenheit an einen Ort, die Notwendigkeit der Anwesenheit der Rezipienten (teilweise auch Produzenten) an einem spezifischen Ort, nicht uneingeschränkt generierbare Rezeptionsräume und zeitgleich begrenzt mögliche Rezipientenzahlen“ sind (2010a: 104). Der Fluchtpunkt von Domkes Beschäftigung mit der Raumgebundenheit von Kommunikation ist die Integration des Raumaspekts in eine Typologie von „Kommunikationsformen“ (vgl. Holly 2000: 83-88, Dürscheid 2005, 2010 oder Schneider 2017). Die verschiedenen Aspekte der Raumgebundenheit, die sie in ihrer Arbeit identifiziert, versteht sie daher als „distinktive[] Merkmal[e]“ (Domke 2010a: 86), die eine feinere Klassifikation von Kommunikationsformen gestatten. ${ }^{32}$

Während der Ansatz der linguistic landscapes im Wesentlichen eine Makroperspektive einnimmt, die die Herstellung von durch Sprachwahl definierten Territorien im Blick hat, ${ }^{33}$ widmen sich die Geosemiotik sowie die zitierten Arbeiten von Auer, Cook, Schmitz, Ziegler und Domke der raumkonstitutiven Wirkung einzelner Texte oder Schilder im Raum. Damit lassen sie sich direkt für die Analyse

32 Mit einem klassifikatorischen Duktus thematisiert auch Adamzik den Einbezug von Raum in die textlinguistische Analyse, und zwar im Rahmen des kurzen Kapitels „Raum-zeitliche Situierung und Objektgebundenheit“ (Adamzik 2004: 78-83). Allerdings sind ihre Ausführungen wenig systematisch, und die behandelten Beispiele und Aspekte des Raumbezugs eröffnen nur streiflichtartige Einblicke in Bedeutungseffekte der Text-Raum-Beziehung.

33 Erst in jüngerer Zeit geraten auch Innenräume in den Blick; s. etwa Karlander (2019) oder Al Saif/Starks (2021). 
der Texte im Ausstellungsraum fruchtbar machen. Allerdings gilt es, die Analysen immer wieder im Moment der Rezeption zu verankern, also etwa die Textfunktionen nicht als etwas zu verstehen, was sich ,automatisch“ aus dem Text und seiner objektiven Position im Raum ergibt. Vielmehr gilt es, von der konkreten Lektüresituation auszugehen (s. Kesselheim 2018, Hausendorf et al. 2017: 84-96) und empiriebasiert danach zu fragen, wie die Rezipienten ausgehend von dem, was sie im Raum wahrnehmen und lesen können, auf eine spezifische Funktion schließen.

\subsubsection{Wissenskommunikation}

Sprachliche Aspekte der Darstellung und Vermittlung von Wissen sind von der Fachsprachenforschung schon lange untersucht worden. In diesem Rahmen wurde ein differenziertes Instrumentarium zur Beschreibung von Fachsprache(n) entwickelt und eine Reihe von Spezifika formuliert, die Fachsprache - und in besonderem Maß die Sprache der Wissenschaft - von der Gemeinsprache unterscheiden (s. den hervorragenden Überblick in Steinhoff 2007: 9-19).

Zur Charakterisierung der Wissenschaftssprache wurden die Begriffe Eindeutigkeit, Exaktheit und Kontextunabhängigkeit vorgeschlagen. Zusätzlich zu diesen grundlegenden Begriffen führt Gloning (2018: 344) „Öffentlichkeit“ und „Kritisierbarkeit“ auf und Janich (2020: 149-151) fügt „intersubjective transparency“, „pleasing aesthetic form“, und „scientific integrity“ als Beschreibungskategorien hinzu.

Die Fachsprachenforschung konzentrierte sich lange Zeit auf den Aspekt der Fachlexik, konkret auf die Frage, welche Merkmale Fachwörter und Wörter der Gemeinsprache voneinander unterscheiden. Untersucht wurde weiterhin, wie Fachwörter in Fachwortschätze bzw. Terminologien eingebettet sind. Erst später wurden nach und nach alle Ebenen der Systemlinguistik in die Analysen einbezogen (s. etwa Möhn/Pelka 1984) und fachsprachentypische Phänomene auf morphologischer Ebene (beispielsweise bestimmte Wortbildungsprozesse) oder syntaktischer Ebene (Satzlänge und -komplexität, Passivkonstruktionen) beschrieben.

Die Öffnung der Fachsprachenforschung hin zu variationistischen Ansätzen lenkte den Blick auf die vielfältigen inneren Differenzierungen der Fachsprache. Dies führte zur Formulierung von Modellen, die den Gegenstand Fachsprache in eine Reihe von Schichten oder Dimensionen untergliedern (s. beispielsweise Ischreyt 1965: 38-50, Hoffmann 1976, Hahn 1983, Kalverkämper 1990, Adamzik 1998, Roelcke 2010: 31). Durch die Fokussierung auf unterschiedliche Grade der „Fachlichkeit“ geriet auch die fachexterne Kommunikation in den Blick, also die sprachlichen Charakteristika der Vermittlung von Fachwissen an Laien. Gleich- 
zeitig aber zog die theoretische Beschäftigung mit den Differenzierungsvorschlägen die Aufmerksamkeit von der Erforschung konkreter sprachlicher Formen ab, die für die Fachsprache als Ganze charakteristisch sind. Diese Tatsache beklagt (Steinhoff 2007: 21) zu Recht, wenn er mit Hinblick auf die von Ehlich (1993: 33) so genannte „Alltägliche Wissenschaftssprache“ kritisiert, dieser Gegenstand sei „von der Fachsprachenforschung und der Wissenschaftslinguistik bislang weitgehend vernachlässigt worden“ und beklagt: „In den beiden Bänden des Handbuchs ,Fachsprachen“ [d.i. Hoffmann 1998 und 1999, W.K.] [...] findet sich kein Artikel, der sich mit dieser Thematik gesondert befasst.“

Die Fach- und Wissenschaftssprache wurden geraume Zeit als Einheit auf System-Ebene aufgefasst und untersucht, was den Blick auf ihren Gebrauch im Rahmen von Wissenskommunikation lange verstellt hat. Das ändert sich ab den 1980er Jahren mit dem Aufkommen der Fachtextforschung (Hoffmann 1988, Göpferich 1995; aktuell zu Textsorten der Wissenschaft: Gloning 2018: 346-350) ${ }^{34}$ und der Fachkommunikationsforschung (Hahn 1983, Engberg 2007). Im Zuge dieser Entwicklung geraten zum einen fachspezifische Textmuster in den Blick (sowohl auf mikro- wie auf makrotextueller Ebene) zum anderen auch pragmatische Aspekte, beispielsweise typische Handlungsstrukturen oder die Einbettung von Fachtexten in weitere kommunikative Kontexte. Dabei werden Fragen akut wie die nach der Konstitution von Laien- und Expertenidentitäten, der Behauptung und Durchsetzung von Expertise, der Konstruktion unterschiedlicher Bilder von Wissenschaft und Wissenschaftlichkeit usw. (s. etwa Gülich 1999, Ciapuscio/Kesselheim 2005, Kesselheim/Thörle 2005, Sandig 2007, Garzone 2010 oder Jaworska 2020).

Eine zweite Traditionslinie der Beschäftigung mit der Darstellung und Vermittlung von Fachwissen ist die Erforschung der Popularisierung von Wissenschaft, also - wie eine oft zitierte Definition lautet - „the transmission of scientific knowledge from scientists to the lay public for purposes of edification, legitimation and training“ (Whitley 1985: 3). Diese „Science of Science Communication“ beschäftigt sich in den allermeisten Fällen mit Formen massenmedialer Kommunikation und versteht sich, wie Gottschlig und Kramer beklagen, heute oftmals als „empirically-shaped media effect studies“ (Gottschling/Kramer 2021: 2f.). Zwar ist in Überblicksdarstellungen immer wieder vom Museum die Rede - vgl. etwa die Einleitung zu Leßmöllmann/Gloning (2020) - doch bleibt die Beschäftigung mit dem Museum oft an der Oberfläche.

Zwar ist das Interesse an diesem Fachgebiet in den letzten zehn Jahren gleichsam explodiert (so Kramer/Gottschling 2021: 1f., Bonfadelli et al. 2017,

34 Textlinguistische Fragestellungen sind noch in den Beiträgen zu Spillner (1994) selten. 
Leßmöllmann et al. 2020; Kessler et al. 2020: 243 speziell zum deutschen Sprachraum). Doch gibt es nur sehr wenige Arbeiten zur Popularisierung aus linguistischer Perspektive.

$\mathrm{Zu}$ den linguistischen Arbeiten, die an konkreten sprachlichen Formen oder Strategien der Wissensvermittlung interessiert sind, zählen Niederhauser (1999), der sich ausführlich mit dem populärwissenschaftlichen Umgang mit Fachtermini sowie mit „Techniken der fachexternen Wissenschaftsvermittlung“ wie der „Reduktion der Informationsfülle“ oder der „Reduktion der Informationsdichte“ beschäftigt (und dem Janich 2020: 155 bescheinigt, seine Untersuchung „remains authoritative even today“), sowie Baumann (2006), der eine Reihe von „Stilfiguren“ und „Denkstilfiguren“ aufzählt, die er als das Verständnis fördernd, das Behalten verbessernd, die Übersichtlichkeit steigernd oder die Eindringlichkeit der Fachtextgestaltung erhöhend analysiert (2006: 208-213). Gloning (2018: 350f.) führt eine Reihe von sprachlichen „Aufgaben“ auf, die „einer wissenschaftlichen Darstellungsform angehören“, etwa das Definieren oder das Rechtfertigen einer Methode.

Hinzu kommen systematische Untersuchungen sprachlicher Vermittlungsphänomene wie die von Brünner/Gülich (2002) so genannten „Veranschaulichungsverfahren“, zu denen die Autorinnen „erstens Reformulierungen, Paraphrasierungen, Explizierungen und Erklärungen, zweitens Veranschaulichungen durch sprachliche Bilder unterschiedlicher Art“ zählen (Brünner/ Gülich 2002: 24; s.a. Brünner 2013). Zwar ist das Konzept der Veranschaulichungsverfahren zunächst an Gesprächen entwickelt worden. Es berücksichtigt aber konzeptionell den Einbezug von dauerkommunikativen Formen in die Interaktion (die Autorinnen denken beispielsweise an Schaubilder oder Tabellen) und lässt sich, wie Ciapuscio (2003) belegt, problemlos auf schriftliche Texte übertragen.

Eine eigene Forschungslinie beschäftigt sich mit der Rolle von Metaphern im Prozess der Popularisierung von Wissen, wobei sowohl deren verständnisfördernde Funktion als auch deren unerwünschte ,Nebenwirkungen“ in den Blick der Forschung geraten sind (Liebert 1996, 2020). Zu nennen sind hier beispielsweise Liebert (1996), Biere/Liebert (1997) oder Drewer (2003). Einen wichtigen Kristallisationspunkt für die linguistische Beschäftigung mit Popularisierung von Wissenschaft stellen schließlich die von Gerd Antos und Sigurd Wichter (1999) begründeten Tagungen zur „Transferwissenschaft“ und die daraus entstandene Publikationsreihe Transferwissenschaften dar (beginnend mit Wichter/Antos 2001), die die gesamte Breite der linguistischen Zugänge zur Wissenskommunikation abbilden, mit einem Schwerpunkt auf Arbeiten aus der angewandten Linguistik. 
Sowohl in der Fachsprachenforschung als auch in der Popularisierungsforschung ist eine Wandlung im Verständnis von Wissen und Wissenskommunikation zu beobachten. So setzt sich in der Popularisierungsforschung eine Sicht durch, die Popularisierung nicht mehr als Prozess der „transmission“ versteht, durch den Wissen an fachfremde Rezipienten ,übertragen' wird, sondern zunehmend als Kommunikationsprozess, bei dem die Rezipienten eine aktive Rolle spielen, indem sie das Fachwissen für sich neu konstruieren müssen (s. Jaworska 2020: 271). ${ }^{35}$ Und in der Fachsprachenforschung fordert Kastberg (2007) die Wendung der Fachsprachenforschung hin zur Erforschung der „knowledge communication“, zu der er schreibt:

As ,communication' it is participative (interactive) and the communicative ,positions' converge on the (co-)construction of (specialized) knowledge. (Kastberg 2007: 8)

Mit der Betonung der „(co-)construction“ trifft sich Kastbergs Definition der Wissenskommunikation mit dem Wissenskonzept von gesprächsanalytischen Arbeiten zur lokalen Konstruktion von Wissen, die ich in 4.2.5 vorstellen werde (s. die Beiträge in Dausendschön-Gay 2010 und speziell zum Museum Kesselheim 2010c). Sie berührt sich aber auch mit Untersuchungen, die die Popularisierung von Wissen und Wissenschaft als Gegenstand gesellschaftlicher Aushandlungsprozesse behandeln: Was als Wissen über ein wissenschaftliches Thema gilt, ist aus dieser Sicht Gegenstand und Ergebnis eines gesellschaftlichen Aushandlungsprozesses. Popularisierung ist, wie (Kailer 2003: 332-334) beispielsweise betont, auch ein ,diskursives Ereignis‘.

Damit verschiebt sich der Fokus der Fachsprachen- und Popularisierungsforschung auf den gesellschaftlichen Kontext, in dem die Wissenskommunikation stattfindet, was diese Disziplinen für die Beschäftigung mit dem Public Understanding of Science sensibilisiert (Stifterverband für die deutsche Wissenschaft 1999, Mooney 2010, Feinstein/Allen/Jenkins 2013, Hendriks/Kienhues 2020). Wissenskommunikation erscheint in diesem Zusammenhang nicht als Vermittlung von isolierten Wissenselementen, sondern auch als Vermittlung der grundlegenden Werte und Methoden der Wissenschaft. Dazu gehört auch die Beschäftigung mit der Frage, wie Fachwissen als vorläufig und korrigierbar präsentiert werden kann, nicht als Produkt, sondern als Prozess und als Gegenstand wissenschaft-

35 Ich spreche in dieser Arbeit durchgehend von der Vermittlung von Wissen. Dieser Begriff scheint mir eher als der der Übertragung mit der Vorstellung kompatibel, dass in diesem Prozess Wissen auch auf Rezipientenseite aktiv konstruiert wird. 
licher und gesellschaftlicher Kontroversen (s. dazu die Beiträge in Liebert 2006, besonders Weitze 2006 zur Darstellung von Kontroversen im Museum).

In der vorliegenden Untersuchung gilt es, die Wissenskommunikation ausgehend von diesen vielfach untereinander verbundenen Forschungssträngen zu beschreiben und dabei herauszuarbeiten, wie diese Wissenskommunikation den spezifischen Möglichkeiten und Problemen der Multimodalität und Raumgebundenheit der Museumsausstellung Rechnung trägt.

\subsection{Die Kommunikation durch die Ausstellung als raumgebundene, multimodale Wissenskommunikation}

Welche Implikationen ergeben sich nun aus der Zusammenschau der vorgestellten theoretischen Ansätze für die Analyse der Ausstellungskommunikation? Hier sollen kurz die wichtigsten Punkte angerissen werden, die dann im folgenden Analysekapitel (s.u., 3.2) ausführlich diskutiert werden sollen:

- Die Kommunikation durch die Ausstellung ist eine spezielle Form von Dauerkommunikation. Als solche ist sie nicht an eine Kommunikationssituation gebunden, in der Sprecher und Hörer gleichzeitig anwesend sind. Die Kommunikation durch die Ausstellung findet dann statt, wenn ein Raumnutzer sinnlich wahrnehmbare Bestandteile des Raums (Architektur, Objekte, Schrift usw.) als Mitteilung versteht, das heißt: als sinnliche Erscheinungsformen von Kommunikation. Will man diese kommunikative Nutzung des Raums verstehen, gilt es - analog zur Analyse der Kommunikation mit Texten (Hausendorf et al. 2017: 96-105) -, die Analyse strikt an die körperliche Wahrnehmungssituation während des Museumsbesuchs anzubinden. Das heißt: all das einzubeziehen (aber auch: sich auf das beschränken), was der Raum ,seinen“ Nutzern im Moment der kommunikativen Nutzung an sinnlichen Wahrnehmungen anbietet.

- Damit geraten viele räumlich-gegenständliche Erscheinungsformen von Kommunikation in den Blick, die bisher von der Linguistik nur am Rande - oder gar nicht - als Bestandteile von Kommunikation aufgefasst und untersucht worden sind. Bei der Faszination, die von der Analyse dieser Erscheinungsformen ausgeht, ist es leicht, über die Rolle der Sprache für die Kommunikation durch die Ausstellung hinwegzusehen. Doch werden die Analysen immer wieder darauf hinweisen, wie Sprache mit diesen ,unkartierten' Modi zusammenspielt: wenn z.B. durch Sprache das bloße Nebeneinander von Objekten $\mathrm{zu}$ Themen wie zoologische Taxonomie, zoogeografische Regionen, Erdzeitalter oder Fundort verdichtet wird (s.u. 3.6) oder aus einem 
unspezifischen „Sehen“ (suggeriert durch das Glas der Vitrine, deren Innenbeleuchtung usw.) ein „Sehen als“ oder ein „Erkennen“ wird (s.u. 3.7).

- Gleichzeitig bedeutet das Ausgehen von der Wahrnehmungssituation, dass die Analysen möglichst spät auf das Vorwissen der Raumnutzer rekurrieren (und dann das Vorwissen an konkrete, sinnlich wahrnehmbare Erscheinungsformen im Raum anbinden, die die Aktivierung dieses Vorwissens konkret nahelegen). Dadurch mag der Eindruck entstehen, dass die Rolle des Vorwissens in den Analysen zu wenig berücksichtigt oder gar ignoriert wird. Tatsächlich ermöglicht die analytische Orientierung auf das Wahrnehmbare aber, Aspekte des Funktionierens der Kommunikation durch die Ausstellung im Detail zu beschreiben, die sonst allzu schnell mit unseren Alltagsgewissheiten zugedeckt worden wären (,Man weiß ja, wo man sich hinstellen soll, um eine Vitrine zu betrachten', s.u. 3.3).

- Schließlich heißt „Ausgehen von der Wahrnehmungssituation“ auch, den Besuchern eine aktive Rolle bei der Konstruktion der Ausstellungsbedeutung zuzugestehen. Bedeutungen sind nicht in die Ausstellung ,eingeschrieben', sie werden von den Besuchern aktiv konstruiert. Dann, wenn sie den Raum daraufhin befragen, welche seiner Elemente wie genau zum raumgebundenen, multimodalen Kommunikationsangebot beitragen (und welche vielleicht nicht dazugehören), wenn sie Verbindungen zwischen raumgebundenen Zeichen erschließen, Antworten auf die Frage suchen, wovon im Raum die Rede ist oder welche kommunikativen Ziele mit dem im Raum arrangierten Kommunikationsangebot verbunden sein mögen (s.u. 3.2.6 zum Konzept der „kommunikativen Aufgaben“ des Ausstellungsraums).

- Bei all dem geht es jedoch nicht um individuelle Besucher und die Art und Weise, wie sie (an einem bestimmten Tag, zu einem bestimmten Zweck, in einer bestimmten Besucherkonstellation usw.) die Bedeutung des Ausstellungsraums konstruiert haben. Ziel der Analyse ist es, Bedeutungspotenziale im Ausstellungsraum zu identifizieren, an denen Besucher (s.o. 1.2.1.3 zum Konzept des „Modell-Besuchers“) anknüpfen, wenn sie den Raum wie selbstverständlich im Sinne der institutionellen Kommunikation nutzen. Tatsächliche Besuchsvorgänge und die interaktiven Prozesse der Bedeutungskonstitution, die in diesen ablaufen, werden Gegenstand von Kapitel 5 und 6 sein. 